\title{
Impulse-driven Richtmyer-Meshkov instability in Hall-magnetohydrodynamics
}

\author{
Naijian Shen $\odot,{ }^{1}$ D. I. Pullin, ${ }^{1}$ Vincent Wheatley, ${ }^{2}$ and Ravi Samtaney ${ }^{3}$ \\ ${ }^{1}$ Graduate Aerospace Laboratories, California Institute of Technology, Pasadena, California 91125, USA \\ ${ }^{2}$ School of Mechanical and Mining Engineering, University of Queensland, QLD 4072, Australia \\ ${ }^{3}$ Mechanical Engineering, Physical Science and Engineering Division, King Abdullah University of Science \\ and Technology, Thuwal 23955-6900, Saudi Arabia
}

(Received 30 July 2019; published 11 October 2019)

\begin{abstract}
We utilize the incompressible, Hall-magnetohydrodynamics (MHD) model for conducting fluids to investigate the effect of Hall current on the stability of an impulsively accelerated, perturbed density interface, or contact discontinuity (CD) separating two fluids in the presence of a background magnetic field. This is used as a simple model, in a conducting fluid, of a Richtmyer-Meshkov type flow that is characterized in a neutral fluid by a shock-wave-density-interface interaction. Two versions of the Hall-MHD equations are explored. In the first, the ions are treated as an incompressible fluid but the electron gas retains its compressibility, while for the second version the incompressible limit for both species is invoked. The linearized equations of motion are first formulated for a sinusoidal interface perturbation and then solved as an initial-value problem using a Laplace transform method with general numerical inversion but with analytical inversion for some limitingparameter cases. While the field equations are identical for both Hall-MHD models, the CD-jump conditions differ leading to qualitatively similar but quantitatively different $C D$ dynamics. For both models, the presence of the magnetic field is found to suppress the incipient interfacial growth associated with neutral-gas, Richtmyer-Meshkov instability (RMI). When the ion skin depth is finite, the vorticity dynamics that drive the suppression of the RMI differ markedly from the ideal MHD, RMI flow. On the interface, the HallMHD description allows the presence of a tangential slip velocity which leads to finite circulation deposition. Away from the interface, vorticity is produced by the perturbed magnetic fields and transported to infinity by a dispersive wave system. This leads to decay of the velocity slip at the interface with the effect that interface growth remains bounded but distorted by damped oscillations associated with the ion cyclotron effect. The flow behavior for several limits of the ion skin depth and the Larmor radius is explored. Specific comparisons with the results from both models against ideal MHD are presented.
\end{abstract}

DOI: 10.1103/PhysRevFluids.4.103902

\section{INTRODUCTION}

First introduced by Markstein [1], before it was studied theoretically by Richtmyer [2] and experimentally by Meshkov [3], the Richtmyer-Meshkov instability (RMI) refers to the growth of perturbations to an interface separating neutral fluids of different densities, typically due to a shock wave traversing the interface. The RMI is pertinent to a wide range of applications, including stellar evolution models in astrophysics [4], shock-flame interactions in combustion systems [5], mixing phenomena in supersonic jet engines [6], and more as surveyed in the review of Brouillette [7]. A richer literature is motivated by inertial confinement fusion (ICF), where a spherically converging shock is driven into a target capsule containing fuel, generating, in principle, conditions at the center sufficient to initiate fusion. The RMI, however, produces mixing between the capsule material and 

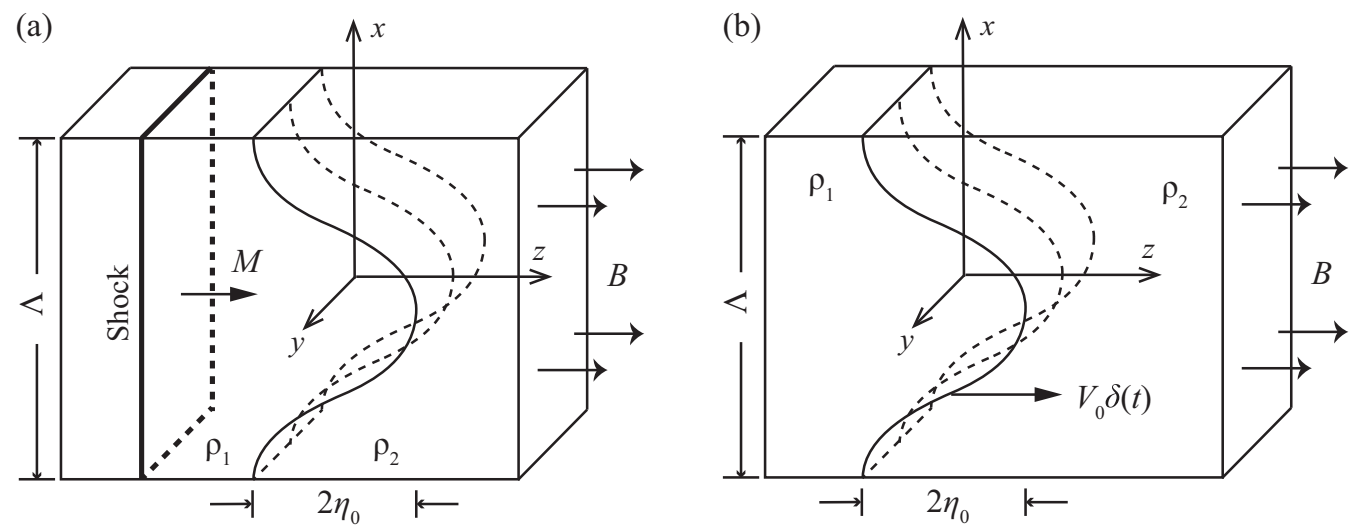

FIG. 1. (a) Geometry and initial condition for compressible RMI with an external magnetic field. (b) The incompressible flow model that mimics the shock with an impulsive acceleration.

the fuel within, limiting final compression and hot-spot production, and thus the ability to achieve energy output [8].

The extreme temperatures required for ICF implosion inevitably causes rapid ionization of the involved materials, which then leads to interaction between the conducting fluids and magnetic fields that are imposed or self-generated [9-12]. In order to model the coupled evolution of plasmas and magnetic fields, several theoretical descriptions have been considered. Samtaney [13] and Wheatley, Samtaney, and Pullin [14,15] employed the single-fluid ideal magnetohydrodynamic (MHD) system to show that the growth of the RMI is suppressed in the presence of a magnetic field normal to the interface. Cao et al. [16] and Wheatley et al. [17] demonstrated that the suppression also occurs for a tangentially applied magnetic field, under the MHD scheme. Srinivasan and Tang [18] adopted the Hall-MHD model to examine the magnetic field generation and growth for the gravity induced Rayleigh-Taylor instabilities (RTI). More recently, Bond et al. [12] investigated computationally the RMI without initial magnetic field using the ideal two-fluid plasma equations. In order of decreasing complexity, Shen et al. [19] showed that the ideal two-fluid plasma equations, the Hall-MHD and regular MHD models, are connected via a series of limiting processes with respect to the appropriately scaled parameters including the speed of light, the ion skin depth, and ion-to-electron mass ratio.

The present study concentrates on the Hall-MHD system, which is a reduced single-fluid model applicable in the regime where the speed of light is large compared to particle thermal speeds and the electron mass is negligible compared to the ion mass. Such system is categorized as a magnetohydrodynamic model for its property of charge neutrality due to effectively infinite speed of light [19]. Nevertheless, the Hall-MHD model captures some features of the more general two-fluid plasma equations over small length scales, such as the Larmor radius and skin depth for ions, that are missing in the ideal MHD equations. Under the Hall-MHD framework, we are interested in the effect of a magnetic field on the RMI flow resulting from a shock wave accelerating a density interface with a single-mode sinusoidal perturbation in amplitude. This is illustrated in Fig. 1(a), where the 2D interface defines a contact discontinuity (CD) between fluids of densities $\rho_{1}$ and $\rho_{2}$, that is to be processed by an incident normal shock of Mach number $M$, traversing in the same direction of an external uniform magnetic field of strength $B$. Cartesian coordinates are assigned so that the unperturbed interface lies in the $x, y$ plane and the single-mode perturbation of wavelength $\Lambda$ and amplitude $\eta_{0}$ varies its magnitude along the $x$ direction.

A more convenient approach, enabling analytic solutions, is to consider an incompressible model for the mass and momentum transport that mimics the shock driven acceleration by an impulse $[14,20]$. Since the electrons are not responsible for the mass transport in Hall-MHD, we consider 
incompressible flow for the ions throughout this paper. The impulse is depicted in Fig. 1(b), where the leading-order effect of the shock is approached by the acceleration, $V_{0} \delta(t)$, where $V_{0}$ matches the postshock interface velocity that would result in a full RM flow and $\delta(t)$ is the Dirac delta function of time $t$. The dynamic response to such an impulse is investigated using two models: first the incompressible ion, compressible electron (IICE) Hall-MHD, and second the incompressible ion, incompressible electron (IIIE) Hall-MHD.

This paper is structured as follows: Section II first derives the general solutions to the linearized IICE Hall-MHD equations, using a weak formulation. The Rankine-Hugoniot conditions for a contact discontinuity specific to the Hall-MHD system are then discussed in order to uniquely close the problem. Section III explores the suppression mechanism for the RMI due to the presence of the magnetic field by examining the vorticity dynamics and performing asymptotic analysis for the IICE system. The alternative IIIE Hall-MHD model is introduced and analyzed in Sec. IV. Illustrative results for the interface behavior and flow profiles are given for both models in Sec. V, before conclusions are drawn in Sec. VI.

\section{INCOMPRESSIBLE ION COMPRESSIBLE ELECTRON (IICE) HALL-MHD}

\section{A. Governing equations}

We begin with the flow model where the density interface perturbed by a single sinusoidal mode is impulsively accelerated to speed $V_{0}$ in the $z$ direction, due to the forcing given by

$$
\boldsymbol{f}=\left[\rho_{1}+H(z)\left(\rho_{2}-\rho_{1}\right)\right] V_{0} \delta(t) \hat{\boldsymbol{z}},
$$

where $H(z)$ is the Heaviside function and $\hat{z}$ is the unit vector in $z$ direction. This leads to a convenient noninertial reference frame that has acceleration $V_{0} \delta(t) \hat{z}$. A connection with a shock-generated Richtmyer-Meshkov flow can be made by identifying the present impulsive velocity $V_{0}$ with the velocity imparted to the $\mathrm{CD}$ by a shock-CD impact. This can be analyzed by the solution to a suitable Riemann-type problem.

Unless otherwise specified, all variables are henceforth made dimensionless by choosing length scale $\Lambda$, velocity scale $V_{0}$, magnetic field scale $B$, charge scale the proton charge $e$, mass scale the ion mass $m_{i}$, and mass density scale $\rho_{1}$. These also lead to the derived reference quantities including the time scale $\Lambda / V_{0}$, the particle number density scale $\rho_{1} / m_{i}$, the pressure scale $\rho_{1} V_{0}^{2}$, the electric field scale $V_{0} B$, and the electric current scale $e \rho_{1} V_{0} / m_{i}$. This normalization scheme arises naturally from the geometry and initial conditions of the present problem, but it differs from the two-fluid plasma RMI study by Bond et al. [12], where the physical Larmor radius and Debye length are used to deduce reference scales for the magnetic field and fluid density.

As a result, one obtains the following nondimensional ideal Hall-MHD equations [19] that govern the evolution of the initial impulse for incompressible ions and compressible electrons,

$$
\begin{aligned}
& \nabla \cdot \boldsymbol{u}=0, \\
& \rho\left(\frac{\partial \boldsymbol{u}}{\partial t}+\boldsymbol{u} \cdot \nabla \boldsymbol{u}\right)=-\nabla p+\frac{1}{d_{L}} \boldsymbol{j} \times \boldsymbol{B}+\boldsymbol{f}-\rho \delta(t) \hat{z}, \\
& \frac{\partial p_{e}}{\partial t}+\left(\boldsymbol{u}-\frac{\boldsymbol{j}}{\rho}\right) \cdot \nabla p_{e}+\gamma p_{e} \nabla \cdot\left(\boldsymbol{u}-\frac{\boldsymbol{j}}{\rho}\right)=0, \\
& \frac{\partial \boldsymbol{B}}{\partial t}+\nabla \times \boldsymbol{E}=\mathbf{0}, \\
& \boldsymbol{E}+\boldsymbol{u} \times \boldsymbol{B}=\frac{\boldsymbol{j} \times \boldsymbol{B}}{\rho}-\frac{d_{L}}{\rho} \nabla p_{e}, \\
& \nabla \times \boldsymbol{B}=\frac{\beta}{2 d_{L}} \boldsymbol{j}, \\
& \nabla \cdot \boldsymbol{B}=0,
\end{aligned}
$$


where $\boldsymbol{u}=(u, v, w)$ is the flow velocity, $\rho$ is the mass density, $\boldsymbol{j}$ is the electric current density, $p$ is the total ion and electron pressure, $p_{e}$ is the electron pressure, $\gamma$ is the specific heat ratio, $\boldsymbol{E}$ is the electric field, and $\boldsymbol{B}$ is the magnetic field. Two dimensionless parameters are introduced, namely the normalized Larmor radius $d_{L}$ and the energy ratio $\beta$, defined as

$$
d_{L}=\frac{V_{0} m_{i}}{e B \Lambda}, \quad \beta=\frac{2 \mu_{0} \rho_{1} V_{0}^{2}}{B^{2}} .
$$

The Larmor radius $d_{L}$ is the normal radius of the helix along which an ion moves about background magnetic field lines while $\beta$ measures the ratio of kinetic to magnetic energy. Following Shen [19], it is useful to define one more related parameter, the normalized ion skin depth $d_{S}$, given by

$$
d_{S}=d_{L} \sqrt{\frac{2}{\beta}},
$$

which is independent of the applied magnetic field strength. Moreover, the regular MHD equations are retrieved by taking the limit of $d_{S} \rightarrow 0$ of the Hall-MHD system [19]. Clearly for any fixed $\beta$, the MHD limit is equivalently achieved by taking $d_{L} \rightarrow 0$. The limit $\beta \rightarrow \infty$ recovers strictly hydrodynamic flow.

\section{B. Linearized system}

We first obtain a base flow solution to (2) that corresponds to the impulsive acceleration of an unperturbed interface. This base flow has no $x, y$ dependence and, owing to the choice of reference frame, the only nonzero vector is the external constant magnetic field. Denoted by the bar symbol, the base flow is thus given by

$$
\begin{aligned}
& \overline{\boldsymbol{u}}=\overline{\boldsymbol{j}}=\overline{\boldsymbol{E}}=\mathbf{0}, \quad \overline{\boldsymbol{B}}=\hat{z}, \\
& \bar{\rho}(z)=\rho_{1}+H(z)\left(\rho_{2}-\rho_{1}\right), \\
& \bar{p}(z, t)=p_{0}, \quad \bar{p}_{e}(z)=p_{e_{0}},
\end{aligned}
$$

where $p_{0}$ and $p_{e_{0}}$ are the constant background total pressure and electron pressure, respectively.

For the perturbed interface, the density profile is expressed as

$$
\rho=\bar{\rho}(z-h),
$$

where $h(x, t)$ is the position of the contact discontinuity and $h \ll 1$ is required to ensure a small perturbation for which linear theory applies. As a result, Eqs. (2) can be linearized around the base flow by perturbing all flow fields using the form

$$
\xi(x, z, t)=\bar{\xi}(z)+\xi^{\prime}(x, z, t),
$$

where $\xi$ generically represents the scalar pressures $p$ and $p_{e}$, or the vector components of $\boldsymbol{u}$ and $\boldsymbol{B} ; \xi^{\prime}$ is the corresponding perturbation of small magnitude. In this formulation we assume no $y$ dependence since the density interface is only perturbed in the $x$ direction without loss of generality.

Using (2f), $\boldsymbol{j}$ is immediately eliminated in terms of $\boldsymbol{B}$. Likewise, taking the curl of (2e) and substituting into (2d) eliminates both $\boldsymbol{E}$ and $p_{e}$, since $\rho$ is constant in each fluid $(z \neq h)$. We note that these reductions are independent of linearization. Therefore, $\bar{p}_{e}$ can be viewed as a decoupled quantity that does not directly affect the magnetofluid dynamics in either fluid. However, its behavior in the vicinity of the interface does play a role in the CD jump conditions, which will be discussed subsequently in Sec. II D. For this consideration, the perturbed electron pressure, $p_{e}^{\prime}$, is explicitly allowed to be discontinuous across the interface by writing

$$
p_{e}^{\prime}(x, z, t)=p_{e_{1}}^{\prime}+H(z-h)\left(p_{e_{2}}^{\prime}-p_{e_{1}}^{\prime}\right) .
$$


As a result, the Hall-MHD system (2) linearizes to give

$$
\begin{aligned}
& \frac{\partial u^{\prime}}{\partial x}+\frac{\partial w^{\prime}}{\partial z}=0, \\
& \rho \frac{\partial u^{\prime}}{\partial t}+\frac{\partial p^{\prime}}{\partial x}=\frac{2}{\beta}\left(\frac{\partial B_{x}^{\prime}}{\partial z}-\frac{\partial B_{z}^{\prime}}{\partial x}\right), \\
& \rho \frac{\partial v^{\prime}}{\partial t}=\frac{2}{\beta} \frac{\partial B_{y}^{\prime}}{\partial z} \\
& \rho \frac{\partial w^{\prime}}{\partial t}+\frac{\partial p^{\prime}}{\partial z}=\left(\rho_{2}-\rho_{1}\right)[H(z)-H(z-h)] \delta(t), \\
& \frac{\partial B_{x}^{\prime}}{\partial t}=\frac{\partial u^{\prime}}{\partial z}+\frac{2 d_{L}}{\beta \rho} \frac{\partial^{2} B_{y}^{\prime}}{\partial z^{2}}, \\
& \frac{\partial B_{y}^{\prime}}{\partial t}=\frac{\partial v^{\prime}}{\partial z}-\frac{2 d_{L}}{\beta \rho} \frac{\partial}{\partial z}\left(\frac{\partial B_{x}^{\prime}}{\partial z}-\frac{\partial B_{z}^{\prime}}{\partial x}\right), \\
& \frac{\partial B_{z}^{\prime}}{\partial t}=\frac{\partial w^{\prime}}{\partial z}-\frac{2 d_{L}}{\beta \rho} \frac{\partial}{\partial z}\left(\frac{\partial B_{y}^{\prime}}{\partial x}\right), \\
& \frac{\partial B_{x}^{\prime}}{\partial x}+\frac{\partial B_{z}^{\prime}}{\partial z}=0,
\end{aligned}
$$

where $B_{x, y, z}^{\prime}$ are the three components of $\boldsymbol{B}^{\prime}$. In particular, a simple auxiliary equation for $p_{e}^{\prime}$ follows from (2a) and (2f),

$$
\frac{\partial p_{e}^{\prime}}{\partial t}=0
$$

Contrasting the strictly two-dimensional MHD case studied by Wheatley et al. [14], here the velocity and magnetic fields must allow nonzero components in the $y$ direction, namely $v^{\prime}$ and $B_{y}^{\prime}$, respectively, for Eqs. (9) to admit nontrivial solutions. Nonetheless, both $v^{\prime}$ and $B_{y}^{\prime}$ in (9) decouple from the system as $d_{L} \rightarrow 0$, and hence the linearized equations converge to those of the MHD case in the limit, as expected [see Eqs. (1)- (5) of Ref. [14]].

To proceed, we make the ansatz that the solutions we are seeking have the following single Fourier-mode form,

$$
\xi^{\prime}(x, z, t)=\tilde{\xi}(z, t) e^{i k x},
$$

where $i$ is the imaginary unit and $k$ is the nondimensional wave number, which takes the value $k=2 \pi$ since a fixed wavelength of $\Lambda$ (dimensional) is used for reference length. The contact is located at $z=h(x, t)$, where

$$
h(x, t)=\eta(t) e^{i k x},
$$

and $\eta(t)$ is the perturbation amplitude.

After substituting (11) into (9), the temporal Laplace transform,

$$
\mathcal{L}[\tilde{\xi}(t)]=\int_{0}^{\infty} \tilde{\xi}(t) e^{-s t} d t, \quad \operatorname{Re}(s)>0,
$$

is further applied in the region $z<0$ and $z>h$, where the impulsive forcing vanishes in (9d). The initial conditions are taken at $t=0^{-}$, just prior to the impulse, when the velocity and magnetic field 
perturbations are zero. As a result, we obtain for each fluid in the region subscripted by $i=1$ or 2 a system of ordinary differential equations (ODEs) in the Laplace space given by

$$
\begin{aligned}
& i k U_{i}+\frac{d W_{i}}{d z}=0, \\
& s \rho_{i} U_{i}+i k P_{i}=\frac{2}{\beta}\left(\frac{d H_{x i}}{d z}-i k H_{z i}\right), \\
& s \rho_{i} V_{i}=\frac{2}{\beta} \frac{d H_{y_{i}}}{d z}, \\
& s \rho_{i} W_{i}+\frac{d P_{i}}{d z}=0, \\
& s \rho_{i} H_{x i}=\rho_{i} \frac{d U_{i}}{d z}+\frac{2 d_{L}}{\beta} \frac{d^{2} H_{y_{i}}}{d z^{2}}, \\
& s \rho_{i} H_{y_{i}}=\rho_{i} \frac{d V_{i}}{d z}-\frac{2 d_{L}}{\beta}\left(\frac{d^{2} H_{x i}}{d z^{2}}-i k \frac{d H_{z i}}{d z}\right), \\
& s \rho_{i} H_{z i}=\rho_{i} \frac{d W_{i}}{d z}-\frac{2 d_{L}}{\beta} i k \frac{d H_{y_{i}}}{d z}, \\
& i k H_{x i}+\frac{d H_{z i}}{d z}=0,
\end{aligned}
$$

where $U, V, W, H_{x}, H_{y}, H_{z}$, and $P$ are the Laplace transforms for $\tilde{u}, \tilde{v}, \tilde{w}, \tilde{B_{x}}, \tilde{B_{y}}, \tilde{B_{z}}$, and $\tilde{p}$, respectively.

Importantly, substituting the Fourier ansatz (11) of $p_{e}^{\prime}$ into (10) implies that for all $z \neq h$ and $t>0$,

$$
\tilde{p}_{e}(z, t)=\tilde{p}_{e}\left(z, 0^{-}\right)=0 .
$$

Therefore, the electron pressure field remains constant, i.e.,

$$
p_{e}=p_{e_{0}} .
$$

\section{General solution}

Equations (14) can be reduced to a single sixth order ODE for $W_{i}$, given by

$$
\mathcal{F}_{i}\left[\left(\frac{d^{2}}{d z^{2}}-k^{2}\right) W_{i}(z)\right]=0,
$$

where $\mathcal{F}_{i}, i=1,2$, is the operator defined for an arbitrary function of $z$ as follows:

$$
\mathcal{F}_{i}=4\left(1+d_{L}^{2} s^{2}\right) \frac{d^{4}}{d z^{4}}-4 s^{2}\left(k^{2} d_{L}^{2}+\beta \rho_{i}\right) \frac{d^{2}}{d z^{2}}+s^{4} \beta^{2} \rho_{i}^{2} .
$$

The fact that (17), derived for the present Hall-MHD model, is of order six is fundamentally different from the regular MHD case where its corresponding ODE for $W_{i}$ is fourth order [see Eq. (12) in Ref. [14]]. Importantly, an order reduction cannot be realized by taking the limit $d_{L} \rightarrow 0$ of (17), where the highest derivative in $\mathcal{F}$ persists. However, $\lim _{d_{L} \rightarrow 0} \mathcal{F}$ does factorize to produce a repeated root, rendering the ODE for $W_{i}$ associated with the regular MHD model a subset of (17) and therefore sufficiently satisfied. This increased order of the present Hall-MHD model is a direct consequence of the velocity and magnetic fields having a self-generated $y$ component, whose significance is discussed also in Sec. II D, where the physical interface boundary conditions required for the Hall-MHD equations are addressed. 
The general solution to (17) is composed of six linearly independent exponentials with coefficients to be determined from appropriate boundary conditions, a procedure that inevitably requires knowledge of higher derivatives of $W_{i}$. However, as we will see later, it is not straightforward to access derivatives of $W_{i}$ from the interface boundary conditions. Therefore, we take a different approach to solve Eq. (14).

Using the definition of $\mathcal{F}$, it is easy to verify that (14) implies the following relations:

$$
\begin{gathered}
\mathcal{F}_{i}\left[s H_{z i}-\frac{d W_{i}}{d z}\right]=0, \\
\mathcal{F}_{i}\left[s H_{x i}-i k W_{i}\right]=0, \\
\mathcal{F}_{i}\left[H_{y_{i}}\right]=0, \\
\mathcal{F}_{i}\left[s \rho_{i} \frac{d W_{i}}{d z}+k^{2} P_{i}\right]=0 .
\end{gathered}
$$

Equation (22), as a consequence of (20) and (21), immediately gives

$$
Q_{i} \equiv s \rho_{i} \frac{d W_{i}}{d z}+k^{2} P_{i}=A_{i} e^{-\mu_{i} z}+B_{i} e^{\mu_{i} z}+C_{i} e^{-\lambda_{i} z}+D_{i} e^{\lambda_{i} z},
$$

where $A_{i}, B_{i}, C_{i}, D_{i}$ are coefficients to be determined and

$$
\begin{aligned}
& \lambda_{i}=\sqrt{\frac{s^{2}\left(\beta \rho_{i}+k^{2} d_{L}^{2}\right)+d_{L} \sqrt{s^{4}\left(2 \beta k^{2} \rho_{i}-\beta^{2} s^{2} \rho_{i}^{2}+k^{4} d_{L}^{2}\right)}}{2 d_{L}^{2} s^{2}+2}}, \\
& \mu_{i}=\sqrt{\frac{s^{2}\left(\beta \rho_{i}+k^{2} d_{L}^{2}\right)-d_{L} \sqrt{s^{4}\left(2 \beta k^{2} \rho_{i}-\beta^{2} s^{2} \rho_{i}^{2}+k^{4} d_{L}^{2}\right)}}{2 d_{L}^{2} s^{2}+2}},
\end{aligned}
$$

are the eigenvalues of the ODE system. Here we choose the branch of the square root that returns positive real part. The boundedness of $Q_{i}$ at $z= \pm \infty$ immediately requires that $A_{1}=B_{2}=C_{1}=$ $D_{2}=0$.

Next, we observe that substituting (14d) into (23) yields

$$
\frac{d^{2} P_{i}}{d z^{2}}-k^{2} P_{i}=-Q_{i},
$$

while using (14a)-(14c) and (14h), together with (23), leads to

$$
\frac{d^{2} H_{z i}}{d z^{2}}-k^{2} H_{z i}=\frac{\beta}{2} Q_{i}
$$

Therefore, taking the general expression for $Q_{i}$ found in (23), $P_{i}$ and $H_{z i}$ can be solved exactly to give

$$
P_{i}(z)=E_{i} e^{-k|z|}+I_{i}, \quad H_{z i}(z)=F_{i} e^{-k|z|}-\frac{\beta}{2} I_{i}
$$

where $E_{i}$ and $F_{i}$ are new coefficients to be determined and $I_{i}$ are the following particular integrals:

$$
I_{1}=\frac{B_{1} e^{\mu_{1} z}}{k^{2}-\mu_{1}^{2}}+\frac{D_{1} e^{\lambda_{1} z}}{k^{2}-\lambda_{1}^{2}}, \quad I_{2}=\frac{A_{2} e^{-\mu_{2} z}}{k^{2}-\mu_{2}^{2}}+\frac{C_{2} e^{-\lambda_{2} z}}{k^{2}-\lambda_{2}^{2}} .
$$

Substituting (23) and (27) into (19) suggests that the coefficients $E_{i}$ and $F_{i}$ are necessarily related,

$$
k^{2} E_{i}+s^{2} \rho_{i} F_{i}=0
$$


With exact solutions for $P_{i}$ and $H_{z i}$ now found, all other variables follow directly from (14), giving

$$
\begin{aligned}
W_{i}(z) & =-\frac{1}{s \rho_{i}} \frac{d P_{i}}{d z}, \quad U_{i}(z)=\frac{i}{k} \frac{d W_{i}}{d z}, \quad V_{i}(z)=\frac{i\left(s H_{z i}-d W_{i} / d z\right)}{k s d_{L}}, \\
H_{x i}(z) & =\frac{i}{k} \frac{d H_{z i}}{d z}, \quad H_{y_{i}}(z)=\frac{1}{s} \frac{d V_{i}}{d z}-\frac{2 d_{L}}{s \beta \rho_{i}}\left(\frac{d^{2} H_{x i}}{d z^{2}}-i k \frac{d H_{z i}}{d z}\right) .
\end{aligned}
$$

\section{Interface jump conditions}

With general solutions to Eqs. (14) to hand, the task remains to determine the six unknown coefficients $A_{2}, B_{1}, C_{2}, D_{1}, F_{1}, F_{2}$. This requires a dedicated discussion of the appropriate jump conditions across the contact discontinuity. For an adiabatically compressible Hall-MHD flow, Hameiri [21] derived in detail the general solvability conditions to support both shocks and CDs. Unlike the regular MHD model, the Hall-MHD shock jump conditions could be nonlocal, depending on the topology of the discontinuity surface and magnetic field. However, for the present investigation with planar density interface and a uniform magnetic field applied normal to the interface, Hameiri's global solvability constraints reduce to the usual Rankine-Hugoniot conditions, obtained by Rosenau et al. [22], from a set of local conservation laws. We adapt the derivation in the following.

The Hall-MHD evolution equations (2) can be formulated weakly in integral form as a system of conservation laws. In addition to (2a) and (2g), which are already in the divergence form, Eq. (2b) in the laboratory frame is equivalent to

$$
\frac{\partial(\rho \boldsymbol{u})}{\partial t}+\nabla \cdot\left[\rho \boldsymbol{u} \boldsymbol{u}+\left(p+\frac{1}{\beta} B^{2}\right) \boldsymbol{I}-\frac{2}{\beta} \boldsymbol{B} \boldsymbol{B}\right]=0 .
$$

It was remarked earlier that the electron pressure cannot affect the flow dynamics in each fluid [21-23]. However, taking the curl of (2e) formally turns (2d) into

$$
\frac{\partial \boldsymbol{B}}{\partial t}+\nabla \cdot\left[\boldsymbol{u} \boldsymbol{B}-\boldsymbol{B} \boldsymbol{u}+\frac{\boldsymbol{B} \boldsymbol{j}-\boldsymbol{j} \boldsymbol{B}}{\rho}\right]=d_{L} \boldsymbol{\nabla} \times\left(\frac{\nabla p_{e}}{\rho}\right),
$$

where the body force term is generally nonzero. However, under the present IICE model where the electron pressure is constant [see Eq. (16)], the body force vanishes across the entire flow domain. This holds particularly at the interface $z=h$, where $\nabla \rho$ diverges. The general consequences of both $\rho$ and $p_{e}$ being discontinuous will be further discussed in Sec. IV B.

To proceed, for an incompressible $\mathrm{CD}$, where the normal flow velocity across the surface vanishes in the interface-stationary reference frame, one must have, across the surface, conservation of mass, momentum, and magnetic fluxes resulting from integrating (2a), (31), (32), and (2g) over a shrinking volume that encloses the surface of discontinuity. This yields the following Rankine-Hugoniot conditions:

$$
\begin{aligned}
\llbracket u_{n} \rrbracket & =0, \\
\llbracket\left(p+\frac{1}{\beta} B^{2}\right) \hat{\boldsymbol{n}}-\frac{2}{\beta} B_{n} \boldsymbol{B} \rrbracket & =0, \\
\llbracket \hat{\boldsymbol{n}} \times \frac{\boldsymbol{j} \times \boldsymbol{B}}{\rho}-B_{n} \boldsymbol{u}_{t} \rrbracket & =0, \\
\llbracket B_{n} \rrbracket & =0,
\end{aligned}
$$

where the square brackets denote the difference in quantity between two sides of the interface, i.e., $\llbracket \xi \rrbracket=\xi_{2}-\xi_{1}$. The subscript " $n$ " indicates the vector component normal to the surface and the subscript " $t$ " indicates the two components tangential to the surface. 
It immediately follows from (33) that, provided the magnetic field is not parallel to the interface, i.e., $B_{n} \neq 0$, both the pressure, $p$, and magnetic field, $\boldsymbol{B}$, must be continuous across the $\mathrm{CD}$, whereas the tangential components of current $\boldsymbol{j}_{t}$ and velocity $\boldsymbol{u}_{t}$ can both jump discontinuously, forming a current sheet and shear layer at the interface. This result contrasts the properties of a regular MHD model, where $\boldsymbol{u}_{t}$ must also be continuous if $B_{n} \neq 0$. It is also noted that the continuity of $\boldsymbol{B}$ validates the use of volume integration of Eq. (32) in order to obtain the correct jump condition, because the magnetic flux to be conserved remains uniformly bounded within the integral domain [21].

For our impulsively accelerated flow, the as yet unknown coefficients in Eqs. (27) can now be determined using (33). This is achieved by first linearizing (33) around the base flow given in Eqs. (5) and (6), before the temporal Laplace transform is applied. As a result, the complete set of jump conditions across the interface [at $z=h(x, t)=\eta(t) e^{i k x}$ ] follows:

$$
\begin{aligned}
& \llbracket W \rrbracket_{z=0}=0, \\
& \llbracket H_{z} \rrbracket_{z=0}=0, \\
& \llbracket H_{x} \rrbracket_{z=0}=\llbracket H_{y} \rrbracket_{z=0}=0, \\
& \llbracket U+\frac{2 d_{L}}{\beta \rho} \frac{d H_{y}}{d z} \rrbracket_{z=0}=0, \\
& \llbracket V-\frac{2 d_{L}}{\beta \rho}\left(\frac{d H_{x}}{d z}-i k H_{z}\right) \rrbracket_{z=0}=0, \\
& \llbracket P \rrbracket_{z=0}=\eta_{0}\left(\rho_{2}-\rho_{1}\right) .
\end{aligned}
$$

In particular, the pressure jump condition is obtained by integrating Eq. (9d) over the forcing region, $0<z<h(x, t)$, and retaining terms of leading order in $h$ to yield

$$
\tilde{p}_{2}(0, t)-\tilde{p}_{1}(0, t)=\left(\rho_{2}-\rho_{1}\right) \delta(t) \eta(t),
$$

whose Laplace transform then leads to (34f).

Although Eqs. (34) appear to be overspecified for only six unknown coefficients, Eqs. (34b) and (34d) can be shown as linearly dependent. Therefore, by substituting (27), (30), and (29) into (34), one finds the unique solution to the vector $\left[A_{2}, B_{1}, C_{2}, D_{1}, F_{1}, F_{2}\right]$ as functions of $s$; hence Eqs. (14) are fully solved. The exact expressions for these coefficients are tedious, and are therefore omitted for brevity.

In the limit $d_{L} \rightarrow 0$, Eqs. (34) recover all the jump boundary conditions for the regular MHD system, except that two new constraints are imposed on $H_{y}$ and $V$, which are the two components in the $y$ direction that decouple in the MHD equations. This is precisely the reason that Eq. (17) is a sixth-order ODE, as opposed to its fourth-order MHD counterpart.

\section{ANALYSIS}

The interface response to the impulsive acceleration is the present model for the RichtmyerMeshkov instability in a Hall-MHD flow. For the present incompressible ion model, because the density interface is a material surface, the time derivative of its perturbation amplitude must equal the normal flow velocity at the interface [see Eq. (12)], that is,

$$
\frac{d \eta}{d t}=\mathcal{L}^{-1}[W(z=0 ; s)]
$$

where $\mathcal{L}^{-1}$ denotes the inverse Laplace transform, formally given by the Bromwich integral,

$$
\mathcal{L}^{-1}[G(s)]=\frac{1}{2 \pi i} \int_{r-i \infty}^{r+i \infty} G(s) e^{t s} d s
$$


where $r \in \mathbb{R}$ is greater than any real part of the singularities of the function $G(s)$. Consequently, the interface amplitude is calculated as

$$
\eta(t)=\eta_{0}+\mathcal{L}^{-1}\left[\frac{W(0 ; s)}{s}\right]
$$

Using the exact solution for either $W_{1}$ or $W_{2}$, it can be verified that the initial interface growth rate is

$$
\left.\frac{d \eta}{d t}\right|_{t=0^{+}}=\eta_{0} k \mathcal{A}
$$

where $\mathcal{A} \equiv\left(\rho_{2}-\rho_{1}\right) /\left(\rho_{2}+\rho_{1}\right)$ is the Atwood number. This initial growth rate is the same as in both the MHD [14] and the hydrodynamic [2] cases, a result which is expected since the initial generation of baroclinic vorticity cannot be affected by the presence of the magnetic field. This is better understood in terms of circulation and vorticity dynamics discussed in the next section.

\section{A. Vorticity dynamics}

Owing to the boundary conditions (34d) and (34e), the tangential velocity obtained from the Hall-MHD description is allowed to slip through the interface, which implies circulation generation, particularly in the $x, z$ plane. To leading order, the circulation over a half wavelength (recalling $\Lambda=1, k=2 \pi)$ deposited at the interface is given by

$$
\Gamma_{0}=\left[U_{2}(0)-U_{1}(0)\right] \int_{0}^{\Lambda / 2} e^{i k x} d x=\frac{2 i \Delta U}{k},
$$

where $\Delta U \equiv U_{2}(0)-U_{1}(0)$. Analogous to (39), the initial circulation evaluates as

$$
\gamma_{0}\left(t=0^{+}\right)=\mathcal{L}^{-1}\left[\Gamma_{0}(s)\right]\left(t=0^{+}\right)=4 \eta_{0} \mathcal{A},
$$

as in the MHD case [15]. However, since $\Gamma_{0}(s) \neq 0$ for arbitrary $s$ in general, the circulation deposited does not instantly leave the interface for the Hall-MHD model, as it does in the MHD case. This property strongly affects the suppression mechanism due to the magnetic field for the Richtmyer-Meshkov instability, as will be seen later.

By taking the curl of the momentum equation (2b), the evolution of vorticity, $\omega=\boldsymbol{\nabla} \times \boldsymbol{u}$, after the initial impulse, is governed by

$$
\frac{\partial \boldsymbol{\omega}}{\partial t}+(\boldsymbol{u} \cdot \nabla) \boldsymbol{\omega}=(\boldsymbol{\omega} \cdot \nabla) \boldsymbol{u}+\frac{\nabla \rho \times \nabla p}{\rho^{2}}+\frac{2}{\beta \rho}[\nabla \times(\nabla \times \boldsymbol{B} \times \boldsymbol{B})],
$$

where the baroclinic term explains the initial circulation generated during the impulsive acceleration. Away from the interface, we impose the same linearization (7) and perturbation (11) to rewrite the vorticity amplitude in the form

$$
\tilde{\boldsymbol{\omega}}=\left(-\frac{\partial \tilde{v}}{\partial z},-i k \tilde{w}+\frac{\partial \tilde{u}}{\partial z}, i k \tilde{v}\right)
$$

Equation (42) then becomes

$$
\frac{\partial \tilde{\boldsymbol{\omega}}}{\partial t}=\frac{2}{\beta \rho}\left(-\frac{\partial^{2} \tilde{B}_{y}}{\partial z^{2}},-i k \frac{\partial \tilde{B}_{z}}{\partial z}+\frac{\partial^{2} \tilde{B}_{x}}{\partial z^{2}}, i k \frac{\partial \tilde{B}_{y}}{\partial z}\right) .
$$

Using (9e)-(9g), together with (43), and differentiating (44) with respect to $t$ gives a forced wave equation for the vorticity vector in the smooth regions of the flow,

$$
\frac{\partial^{2} \tilde{\boldsymbol{\omega}}}{\partial t^{2}}=\frac{2}{\beta \rho_{i}} \frac{\partial^{2} \tilde{\boldsymbol{\omega}}}{\partial z^{2}}+\frac{4 d_{L}}{\beta^{2} \rho_{i}^{2}}\left(\frac{\partial^{4} \tilde{\boldsymbol{B}}}{\partial z^{4}}-k^{2} \frac{\partial^{2} \tilde{\boldsymbol{B}}}{\partial z^{2}}\right) .
$$


In the MHD limit, where $d_{L} \rightarrow 0$, the forcing term vanishes and the vorticity propagates in each side of the interface according to the wave equation, with normalized Alfvén speeds $v_{A}=\sqrt{2 /\left(\beta \rho_{i}\right)}$ [19], for $i=1,2$, respectively.

Since circulation is only generated at the interface at $t=0^{+}$for the MHD flow before it is instantly carried away by the Alfvén waves, it is implied that the total circulation, integrated over the $z$ axis, must be conserved over time, as found by Wheatley et al. [15]. In contrast, for the present Hall-MHD flow, where $d_{L}>0$, the forcing term in Eq. (45) is generally nonzero. Indeed, introducing Hall current produces a magnetic field [18], as can be seen from (9e)-(9g), written compactly as

$$
\frac{\partial \boldsymbol{B}^{\prime}}{\partial t}=\frac{\partial \boldsymbol{u}^{\prime}}{\partial z}-\frac{2 d_{L}}{\beta \rho} \nabla \times\left(\nabla \times \boldsymbol{B}^{\prime} \times \hat{z}\right),
$$

which now has a nonzero $y$ component. Additionally, we have shown previously that there is always circulation accumulated on the interface. Therefore, the conservation of total circulation is not expected, which resembles a feature observed numerically for the two-fluid RMI flow [12].

\section{B. Asymptotic analysis}

In this section we explore analytically the behavior of the IICE Hall-MHD flow solution found in Sec. II C for limiting values of the Larmor radius $d_{L}$ and the energy ratio $\beta$.

\section{Small Larmor radius: The MHD limit}

It was previously shown in Sec. II C that the $d_{L} \rightarrow 0$ limit of the linearized incompressible HallMHD system (14) contains the governing equations for the MHD flow [14], and in Sec. II D that the Hall-MHD jump conditions include all those of the MHD equations in this limit. Therefore, the Hall-MHD solutions obtained in Eq. (30) must converge to those in the MHD case. Indeed it is verified that, for any fixed $\beta>0$, the substitution of $d_{L}=0$ into the exact expressions for coefficients $\left[A_{2}, B_{1}, \ldots, F_{2}\right]$ produces a removable singularity. The limiting expression for $W$ at the interface is given by

$$
\lim _{d_{L} \rightarrow 0} W(0 ; s)=\frac{\eta_{0} k\left[4 k^{2}(r-\sqrt{r}) v_{A_{2}}+2 k(\sqrt{r}-1)^{2} s-\sqrt{2 \beta}(r-1) s^{2}\right]}{4 k^{3} r v_{A_{2}}^{2}+4 k^{2} r v_{A_{2}} s+2 k(r-2 \sqrt{r}-1) s^{2}-\sqrt{2 \beta}(r+1) s^{3}},
$$

where the normalization $r \equiv \rho_{2} / \rho_{1}=\rho_{2}$ is used and $v_{A_{2}}=\sqrt{2 / \beta \rho_{2}}$ is the Alfvén speed in the region $i=2$. This result agrees with the MHD solutions found by Wheatley et al. [14]. More interestingly, the $y$ components of the velocity and magnetic fields which are missing in the MHD description decay identically in the limit

$$
\lim _{d_{L} \rightarrow 0} V_{i}(z ; s)=\lim _{d_{L} \rightarrow 0} H_{y_{i}}(z ; s)=0 .
$$

This result validates the strictly two-dimensional flow assumption made by the MHD model [14,15].

The success of recovering the MHD solution at leading order as $d_{L} \rightarrow 0$ usually suggests a first order Hall-MHD correction to the regular MHD theory for small values of $d_{L}$. Nonetheless, the existence of a uniformly valid correction is contradicted by the exponential exponents associated with the general solutions, given in Eqs. (24). Specifically, these exponents $\left(\lambda_{i}\right.$ and $\left.\mu_{i}\right)$ have singular points located at $s= \pm i / d_{L}$; therefore, when expanded as power series around $d_{L}=0$, the radius of convergence of the series decreases to zero as $\operatorname{Im}(s)$ becomes large. Consequently, a uniformly valid approximation of the exponents that is linear in $d_{L}$ cannot exist along the entire Bromwich contour as would be required for accurate calculation of the inverse Laplace transform.

\section{Small plasma beta limit}

Here, we turn our attention to a different flow region where the hydrodynamic forces are dominated by the electromagnetic forces, which corresponds to the asymptotic limit of $\beta \rightarrow 0$. 
Such a limit may be approached while keeping either $d_{S}$ or $d_{L}$ fixed. In the former route where $\beta \rightarrow 0$ with $d_{S}$ fixed, all field variables in (30) decay asymptotically as

$$
W_{i}, V_{i} \sim O\left(\beta^{1 / 2}\right), \quad U_{i}, H_{x_{i}}, H_{y_{i}}, H_{z_{i}} \sim O(\beta),
$$

suggesting that increasing the external magnetic field eventually inhibits all dynamical motions in both flow regions separated by the density interface. In particular, the interface amplitude decays inversely proportional to the applied magnetic field strength for strong field, in agreement with the MHD theory [14].

However, the second route of taking $\beta \rightarrow 0$ while keeping $d_{L}$ fixed produces a nontrivial solution. In this case, the eigenvalues, $\lambda_{i}, \mu_{i}$, and consequently all the coefficients, $\left[A_{2}, B_{1}, \ldots, F_{2}\right]$, can be Taylor expanded about $\beta=0$ to give the leading order expressions:

$$
\begin{aligned}
A_{2} & =\frac{\eta_{0} k^{2}\left(\rho_{1}-\rho_{2}\right)^{2} \rho_{2}}{\rho_{1}^{2}(\alpha+1)+2 \rho_{2} \rho_{1}(\alpha-1)+\rho_{2}^{2}(\alpha+1)}, \\
B_{1} & =\frac{\eta_{0} k^{2} \rho_{1}\left(\rho_{1}-\rho_{2}\right)^{2}}{\rho_{1}^{2}-2 \rho_{2} \rho_{1}+\rho_{2}^{2}+\alpha^{2}\left(\rho_{1}+\rho_{2}\right)^{2}}, \\
C_{2} & =\frac{\eta_{0} k^{2} \rho_{2}\left(\rho_{2}^{2}-\rho_{1}^{2}\right)}{\rho_{1}^{2}\left(\alpha^{2}+\alpha\right)+2 \rho_{2} \rho_{1}\left(\alpha^{2}-\alpha\right)+\rho_{2}^{2}\left(\alpha^{2}+\alpha\right)}, \\
D_{1} & =\frac{\eta_{0} k^{2} \rho_{1}\left(\rho_{1}^{2}-\rho_{2}^{2}\right)}{\rho_{1}^{2}\left(\alpha^{2}+\alpha\right)+2 \rho_{2} \rho_{1}\left(\alpha^{2}-\alpha\right)+\rho_{2}^{2}\left(\alpha^{2}+\alpha\right)}, \\
E_{1} & =E_{2}=F_{1}=F_{2}=0,
\end{aligned}
$$

where $\alpha \equiv \sqrt{d_{L}^{2} s^{2}+1}$.

It then follows from Eq. (30) that, in the Laplace space, the limiting magnetic field perturbations are identically zero, i.e., $H_{x}=H_{y}=H_{z}=0$, while the flow velocities, $U$ and $W$, are given by

$$
\begin{gathered}
U_{1,2}(z)= \pm \frac{i \eta_{0} \mathcal{A} k d_{L}^{2} s}{\alpha\left(\mathcal{A}^{2}+\alpha\right)} \exp \left(-\frac{k d_{L} s}{\alpha}|z|\right), \\
W_{1,2}(z)=\frac{\eta_{0} \mathcal{A} k d_{L}}{\mathcal{A}^{2}+\alpha} \exp \left(-\frac{k d_{L} s}{\alpha}|z|\right) .
\end{gathered}
$$

Thus, using the Laplace final-value theorem, both velocities in the time domain converge to zero. More specifically, at $z=0$, these expressions can be inverse transformed to give the $x, z$-plane circulation and the interface growth rate, using Eqs. (41) and (36), respectively,

$$
\begin{gathered}
\hat{\gamma}_{0}=\cos (\phi T)+\frac{1}{\mathcal{A}^{2}}\left[\phi \sin (\phi T)-\sin (T)+[\cos (T)-\cos (\phi T)] * \frac{J_{1}(T)}{T}\right], \\
\frac{d \hat{\eta}}{d t}=\cos (\phi T)-\frac{\mathcal{A}^{2} \sin (\phi T)}{\phi}+\frac{\sin (\phi T)}{\phi} * \frac{J_{1}(T)}{T},
\end{gathered}
$$

where $\hat{\gamma}_{0} \equiv \gamma_{0} /\left(4 \eta_{0} \mathcal{A}\right), \phi \equiv \sqrt{1-\mathcal{A}^{4}}, T \equiv t / d_{L}, \hat{\eta} \equiv \eta\left(\mathcal{A} \eta_{0} k\right)^{-1}, J_{1}$ is the first order Bessel function of the first kind, and $*$ denotes the convolution integral defined as

$$
f(T) * g(T)=\int_{0}^{T} f(\tau) g(T-\tau) d \tau .
$$

Similarly, using Eq. (38), the limiting interface amplitude in the time domain is given by

$$
\frac{\hat{\eta}(t)-\hat{\eta}_{0}}{d_{L}}=\frac{\phi \sin (\phi T)-\mathcal{A}^{2}[1-\cos (\phi T)]}{\phi^{2}}+\frac{1-\cos (\phi T)}{\phi^{2}} * \frac{J_{1}(T)}{T},
$$


whose long-time behavior follows directly from (52),

$$
\lim _{t \rightarrow \infty} \eta(t)=\eta_{0}\left(1+\frac{\mathcal{A} k d_{L}}{\mathcal{A}^{2}+1}\right) .
$$

In the zero $\beta$ limit with $d_{L}$ fixed, Eqs. (53)-(56) clearly show oscillatory behavior of the flow occurring on a time scale $T$, that is completely specified by the nondimensional Larmor radius $d_{L}$. This phenomenon differs significantly from the regular MHD case. For instance, unlike the MHD theory where the growth rate of the interface amplitude decays monotonically towards zero, the limiting Hall-MHD model exhibits sinusoidal fluctuations with a decaying envelope, which eventually converges as $t \rightarrow \infty$, as seen in Eq. (54). We will see in Sec. V that such behavior can be extrapolated to solutions obtained for nonzero $\beta$.

To explain these periodic motions, the dispersive nature of the Hall-MHD equations is considered. In particular, the Hall-MHD system captures what is missing in the regular MHD description, the ion cyclotron resonance, whose frequency is inversely proportional to $d_{L}[19,24]$, naturally reflecting the new time scale $T$. Therefore, when the dimensional Larmor radius of the ions is comparable to the geometric length scale, namely, $d_{L} \sim O(1)$, the ion gyromotion around the overwhelming magnetic field lines becomes significant, as clearly manifested in the time domain solutions derived in this section, where time is more appropriately scaled by the cyclotron frequency and length is more suitably normalized by the Larmor radius.

\section{Large Larmor radius limit}

Inspired by the cyclotron frequency dependency discovered above, we look here for the asymptotic solution opposite to the MHD description, that is, the $d_{L} \rightarrow \infty$ limit for any constant time, $T=t / d_{L}$, which is rescaled according to the cyclotron frequency. Specifically, this limit is calculated by first applying the change of Laplace variable, $s \mapsto \sigma / d_{L}$. Exact expressions of the eigenvalues, $\lambda_{i}$ and $\mu_{i}$, hence all coefficients, $\left[A_{2}, B_{2}, \ldots, F_{2}\right]$, derived from Eq. (34) as functions of $\sigma$, are then expanded in power series of $\epsilon \equiv 1 / d_{L}$ around $\epsilon=0$. Here, an effective distinguished limit is implied with $t \rightarrow \infty$, while holding $T$ constant. Otherwise, convergent solutions cannot be found for the original time $t$, as $d_{L} \rightarrow \infty$.

Surprisingly, in the infinite Larmor radius limit with appropriate time rescaling, we obtain exactly the same expressions for all coefficients as those given in Eq. (50), except for $F_{1}$ and $F_{2}$, whose asymptotic forms are found in the following:

$$
F_{1}(\sigma)=\frac{\mathcal{A} \beta \eta_{0} \rho_{1}\left(\alpha+\mathcal{A}^{2}-\mathcal{A} \sigma\right)}{2(\mathcal{A}-1)\left(\alpha+\mathcal{A}^{2}\right)}, \quad F_{2}(\sigma)=\frac{\mathcal{A} \beta \eta_{0} \rho_{1}\left(\alpha+\mathcal{A}^{2}+\mathcal{A} \sigma\right)}{2(1-\mathcal{A})\left(\alpha+\mathcal{A}^{2}\right)},
$$

where $\alpha=\sqrt{\sigma^{2}+1}$, as before. Therefore, the interface circulation deposit $\gamma_{0}$ and the rescaled perturbation growth $\eta / d_{L}$ (as a function of $T$ ) in this case are the same as those of the zero plasma beta limit, derived in Eqs. (53) and (56), respectively. Furthermore, nontrivial solutions in the Laplace space to the magnetic field perturbations $H_{x, y, z}$ appear in the $d_{L} \rightarrow \infty$ limit; for example, at the interface, one has

$$
H_{z}(z=0 ; \sigma)=\frac{\mathcal{A}^{2} \beta \eta_{0} \rho_{1}(\alpha-\sigma+1)}{2(\mathcal{A}-1)\left(\alpha+\mathcal{A}^{2}\right)} .
$$

However, the corresponding inverse transform of (59) in the cyclotron time scale $\left(\mathcal{L}^{-1}: \sigma \mapsto T\right)$ again yields a vanishing physical field $\tilde{B}_{z}(T)=O\left(1 / d_{L}\right)$, due to the change of variable $s=\sigma / d_{L}$.

Finally, we remark on the commonalities between the zero beta limit and the infinite Larmor radius limit of the Hall-MHD theory. In both cases, the ion skin depth, $d_{S}$, defined in Eq. (4), approaches infinity, giving the same interface growth and circulation behavior that differs from the MHD prediction, which corresponds to the zero ion skin depth limit of the Hall-MHD model. Moreover, the magnetic field perturbations vanish in both cases, at a rate of $O(\beta)$ as $\beta \rightarrow 0$ or at a rate of $O\left(1 / d_{L}\right)$ in the cyclotron time scale as $d_{L} \rightarrow \infty$. 


\section{INCOMPRESSIBLE ION INCOMPRESSIBLE ELECTRON (IIIE) HALL-MHD}

In this section, we propose a different Hall-MHD model which further assumes that the electrons also behave like an incompressible fluid, consistent with the ions. This leads to a subtle change in the $\mathrm{CD}$ jump conditions which then results in interface behavior different than that analyzed previously for the IICE model.

\section{A. Governing equations and general solutions}

Although the Hall-MHD system is effectively a one-fluid model, it nevertheless allows a reconstruction of separate ion and electron velocity field, $\boldsymbol{u}_{i, e}$, respectively. Recalling $m_{i}=1$ and $e=1$ under the present nondimensionlization scheme, the species flow fields are given by [19,21]

$$
\boldsymbol{u}_{i}=\boldsymbol{u}, \quad \boldsymbol{u}_{e}=\boldsymbol{u}-\frac{\boldsymbol{j}}{\rho}
$$

Therefore, the additional incompressible electron's assumption immediately gives the continuity condition in terms of the current $\boldsymbol{j}$,

$$
\nabla \cdot\left(\frac{j}{\rho}\right)=0
$$

at the cost of losing the dynamic equation for electron pressure, as (2c) in the IICE model. For completeness, the full set of governing equations in the impulsively accelerated frame is now given by

$$
\begin{aligned}
& \nabla \cdot \boldsymbol{u}=0, \quad \nabla \cdot\left(\frac{\boldsymbol{j}}{\rho}\right)=0, \\
& \rho\left(\frac{\partial \boldsymbol{u}}{\partial t}+\boldsymbol{u} \cdot \nabla \boldsymbol{u}\right)=-\nabla p+\frac{1}{d_{L}} \boldsymbol{j} \times \boldsymbol{B}-\rho \delta(t) \hat{\boldsymbol{z}}+\left[\rho_{1}+H(z)\left(\rho_{2}-\rho_{1}\right)\right] \delta(t) \hat{\boldsymbol{z}}, \\
& \frac{\partial \boldsymbol{B}}{\partial t}+\nabla \times \boldsymbol{E}=\mathbf{0}, \quad \boldsymbol{E}+\boldsymbol{u} \times \boldsymbol{B}=\frac{\boldsymbol{j} \times \boldsymbol{B}}{\rho}-\frac{d_{L}}{\rho} \nabla p_{e}, \\
& \nabla \times \boldsymbol{B}=\frac{\beta}{2 d_{L}} \boldsymbol{j}, \quad \nabla \cdot \boldsymbol{B}=0 .
\end{aligned}
$$

Importantly, it is noted that, using system (62) in the smooth flow regions with constant density, the electron pressure $p_{e}$ is indeterminate and the electric field $\boldsymbol{E}$ is found up to a gauge. Indeed, we can define modified variables,

$$
\boldsymbol{E}^{*}=\boldsymbol{E}-\nabla f, \quad p_{e}^{*}=p_{e}+\frac{\rho f}{d_{L}},
$$

using an arbitrary scalar function $f \in C^{1}\left(\mathbb{R}^{3}\right)$ to verify that Eqs. (62) are identically satisfied by the modified variables $\boldsymbol{E}^{*}$ and $p_{e}^{*}$. For $\boldsymbol{E}$ to be uniquely invertible, both of its curl and divergence are needed. However, $\boldsymbol{\nabla} \cdot \boldsymbol{E}$ is related to a small degree of charge separation that is not prescribed in Hall-MHD [19].

Nonetheless, $p_{e}, \boldsymbol{E}$, and $\boldsymbol{j}$ here can be conveniently eliminated as for the IICE model, leaving Eq. (61) automatically satisfied. The resulting simplified system thus becomes identical to Eqs. (9), subject to the same linearization around the base flow given in Sec. II B. Therefore, on each side of the perturbed interface, the general solutions to the flow velocity and magnetic fields derived in Sec. II C are not affected by the additional treatment of incompressible electrons here. Similarly, the vorticity transport mechanism described in Sec. III A applies the same for both of the IICE and IIIE models. 


\section{B. Modified interface jump conditions}

Losing the determinacy of $p_{e}$ in the IIIE model has not yet played a part in the flow dynamics in each fluid away from the density interface. However, as shown in Eq. (32), its behavior near the interface was crucial for the conservation of magnetic flux. Without an independent evolution equation for $p_{e}$ in the present model, one cannot reach the constant electron pressure solution found in (16) for the IICE model. In this case, the forcing term in (32) could be evaluated using (6) and (12) to yield

$$
\boldsymbol{b} \equiv \nabla \times\left(\frac{\nabla p_{e}}{\rho}\right)=\left[\frac{\rho_{1}-\rho_{2}}{\rho^{2}} \delta\left(z-\eta e^{i k x}\right)\left(\frac{\partial p_{e}}{\partial x}+i k \eta e^{i k x} \frac{\partial p_{e}}{\partial z}\right)\right] \hat{\boldsymbol{y}},
$$

where $\hat{\boldsymbol{y}}$ is the unit vector in $y$ direction. Further applying (8) and (11) and integrating across the interface then gives

$$
\int_{h^{-}}^{h^{+}} \boldsymbol{b} \cdot \hat{\boldsymbol{y}} d z=\frac{2 i k\left(\rho_{1}-\rho_{2}\right)}{\left(\rho_{1}+\rho_{2}\right)^{2}}\left[\tilde{p}_{e_{1}}\left(0^{-}, t\right)+\tilde{p}_{e_{2}}\left(0^{+}, t\right)\right] e^{i k x}+O(\eta),
$$

where $H(0)=1 / 2$ for the Heaviside function is chosen.

Consequently, the conservation of magnetic flux across the interface as given in (33c) must be modified to incorporate the finite contribution from the body force. To leading order, Eq. (34e) should change into

$$
\llbracket V-\frac{2 d_{L}}{\beta \rho}\left(\frac{d H_{x}}{d z}-i k H_{z}\right) \rrbracket_{z=0}=\frac{2 i k d_{L}\left(\rho_{1}-\rho_{2}\right)}{\left(\rho_{1}+\rho_{2}\right)^{2}} \mathcal{L}\left[\tilde{p}_{e_{1}}\left(0^{-}, t\right)+\tilde{p}_{e_{2}}\left(0^{+}, t\right)\right] e^{i k x},
$$

which now serves as a constraint on $p_{e}$, which is indeterminate in the present IIIE model.

Since (66) cannot be used effectively as a boundary condition in order to determine the coefficients $\left[A_{2}, B_{1}, C_{2}, D_{1}, F_{1}, F_{2}\right]$, closure must be established from the continuity condition (61), which is exclusive to the IIIE model and is nontrivial only across the interface, giving

$$
\llbracket \frac{j_{n}}{\rho} \rrbracket=\llbracket \frac{1}{\rho} \frac{\partial B_{y}}{\partial x} \rrbracket=0 .
$$

Therefore, as density jumps over $\mathrm{CD}$, the normal component of current density has to vanish. In the Laplace space, this implies at leading order

$$
\llbracket H_{y} \rrbracket_{z=0}=0 .
$$

Equation (67) effectively states that penetration of current across a density interface is prohibited, a direct consequence of both the ions and electrons being incompressible. The reason is that all charged particles for the IIIE model must move at the same velocity as the CD for the latter to evolve as a material surface. In contrast, for the previous IICE model, the electrons are free to move across the $\mathrm{CD}$ without violating conservation of mass in Hall-MHD.

\section{Asymptotic flow field}

Combining Eqs. (34a)-(34c), (34f), and (68) gives a linear system that uniquely determines the coefficients $\left[A_{2}, B_{1}, C_{2}, D_{1}, F_{1}, F_{2}\right]$ for the IIIE model. The complete solution differs from that discussed in Sec. II D. We present its asymptotic form in the same limits as discussed in Sec. III B.

First, it is no surprise that, for a fixed energy ratio $\beta$, taking the $d_{S} \rightarrow 0$ limit recovers the regular MHD solution (see Sec. III B 1) in the IIIE model as well. Indeed the distinguishing boundary condition (68) is already met in the regular MHD model, where the vector fields are genuinely two dimensional in the $x, z$ plane.

Here we focus on the large skin depth limit $\left(d_{S} \rightarrow \infty\right)$, opposite to the regular MHD description, achieved via (a) taking $\beta \rightarrow 0$ while holding $d_{L}$ fixed or (b) letting $d_{L} \rightarrow \infty$ while keeping $\beta$ finite. 
Analogous results to those obtained in Secs. III B 2 and III B 3 follow as

$$
\begin{aligned}
& A_{2}=\frac{\eta_{0} k^{2} \rho_{2}\left(\rho_{2}-\rho_{1}\right)}{\left(\rho_{1}+\rho_{2}\right)(\alpha+1)}, \quad B_{1}=\frac{\eta_{0} k^{2} \rho_{1}\left(\rho_{1}-\rho_{2}\right)}{\left(\rho_{1}+\rho_{2}\right)(\alpha+1)}, \\
& C_{2}=\frac{\eta_{0} k^{2} \rho_{2}\left(\rho_{2}-\rho_{1}\right)}{\left(\rho_{1}+\rho_{2}\right)\left(\alpha^{2}+\alpha\right)}, \quad D_{1}=\frac{\eta_{0} k^{2} \rho_{1}\left(\rho_{1}-\rho_{2}\right)}{\left(\rho_{1}+\rho_{2}\right)\left(\alpha^{2}+\alpha\right)},
\end{aligned}
$$

and

$$
F_{1}=\left\{\begin{array}{ll}
0, & \beta \rightarrow 0, \\
\frac{\mathcal{A} \beta \eta_{0} \rho_{1}(\alpha+1-\mathcal{A} \sigma)}{2(\mathcal{A}-1)(\alpha+1)}, & d_{L} \rightarrow \infty,
\end{array} \quad F_{2}= \begin{cases}0, & \beta \rightarrow 0, \\
\frac{\mathcal{A} \beta \eta_{0} \rho_{1}(\mathcal{A} \sigma+\alpha+1)}{2(1-\mathcal{A})(\alpha+1)}, & d_{L} \rightarrow \infty,\end{cases}\right.
$$

where $\sigma=d_{L} s, \alpha=\sqrt{\sigma^{2}+1}$, and $\mathcal{A}=\left(\rho_{2}-\rho_{1}\right) /\left(\rho_{2}+\rho_{1}\right)$ is the Atwood number, as before. This leads to the limiting in-plane flow field as $d_{S} \rightarrow \infty$ in the Laplace space given by

$$
\begin{gathered}
U_{1,2}(z)= \pm \frac{i \eta_{0} \mathcal{A} k d_{L}^{2} s}{\alpha(1+\alpha)} \exp \left(-\frac{k \sigma}{\alpha}|z|\right), \\
W_{1,2}(z)=\frac{\eta_{0} \mathcal{A} k d_{L}}{1+\alpha} \exp \left(-\frac{k \sigma}{\alpha}|z|\right) .
\end{gathered}
$$

Interestingly, these expressions share the same limits of Eqs. (51) and (52) as $\mathcal{A} \rightarrow 1$, suggesting that the IICE and IIIE models converge when both the skin depth and density ratio become large $\left(d_{S} \rightarrow \infty, \rho_{2} / \rho_{1} \rightarrow \infty\right)$. In this case, the inverse transform $\left(\mathcal{L}^{-1}: \sigma \rightarrow T\right)$ at the interface can be calculated in closed form using binomial expansion to yield

$$
\hat{\gamma}_{0}=\mathcal{L}^{-1}\left[\frac{\sigma}{\alpha+\alpha^{2}}\right]=1-T_{1} F_{2}\left(\frac{1}{2} ; 1, \frac{3}{2} ;-\frac{T^{2}}{4}\right)
$$

and

$$
\begin{gathered}
\frac{d \hat{\eta}}{d t}=\mathcal{L}^{-1}\left[\frac{1}{1+\alpha}\right]={ }_{1} F_{2}\left(-\frac{1}{2} ; \frac{1}{2}, 1 ;-\frac{T^{2}}{4}\right)-T, \\
\frac{\hat{\eta}-\hat{\eta}_{0}}{d_{L}}=\mathcal{L}^{-1}\left[\frac{1}{\sigma(1+\alpha)}\right]=T_{1} F_{2}\left(-\frac{1}{2} ; 1, \frac{3}{2} ;-\frac{T^{2}}{4}\right)-\frac{T^{2}}{2},
\end{gathered}
$$

where ${ }_{1} F_{2}$ is the generalized hypergeometric function [25] and, again, $T=t / d_{L}, \hat{\gamma}_{0}=\gamma_{0} /\left(4 \eta_{0} \mathcal{A}\right)$, and $\hat{\eta}=\eta\left(\mathcal{A} \eta_{0} k\right)^{-1}$.

\section{NUMERICAL RESULTS}

The time-dependent behavior of the velocity, vorticity and magnetic fields, interface growth, and other quantities of interest is retrieved from the $s$-dependent expressions given in Eqs. (27) and (30), for both the IICE and IIIE models, by applying the inverse Laplace transform. For general parameter values of the Larmor radius $d_{L}$ and the energy ratio $\beta$, this is performed numerically for a given time, $t$, using the multiprecision Talbot method [26], originally proposed by Talbot [27]. The algorithm involves deforming the Bromwich contour [see Eq. (37)] using the parametrization

$$
s(\theta)=\frac{2 N}{5 t} \theta(\cot \theta+i), \quad-\pi<\theta<\pi,
$$

where $N$ is the truncation number used in the trapezoidal rule to numerically evaluate the deformed integral. $N$ also specifies the number of precision decimal digits in a symbolic environment, such as mathematica ${ }^{\circledR}$, where the Talbot algorithm is implemented. Convergent results are obtained by gradually increasing $N$ up to $N=300$. Particularly, the numerical solutions for the limiting parameters match those given by the exact asymptotic expressions available in Secs. III B and IV C. 

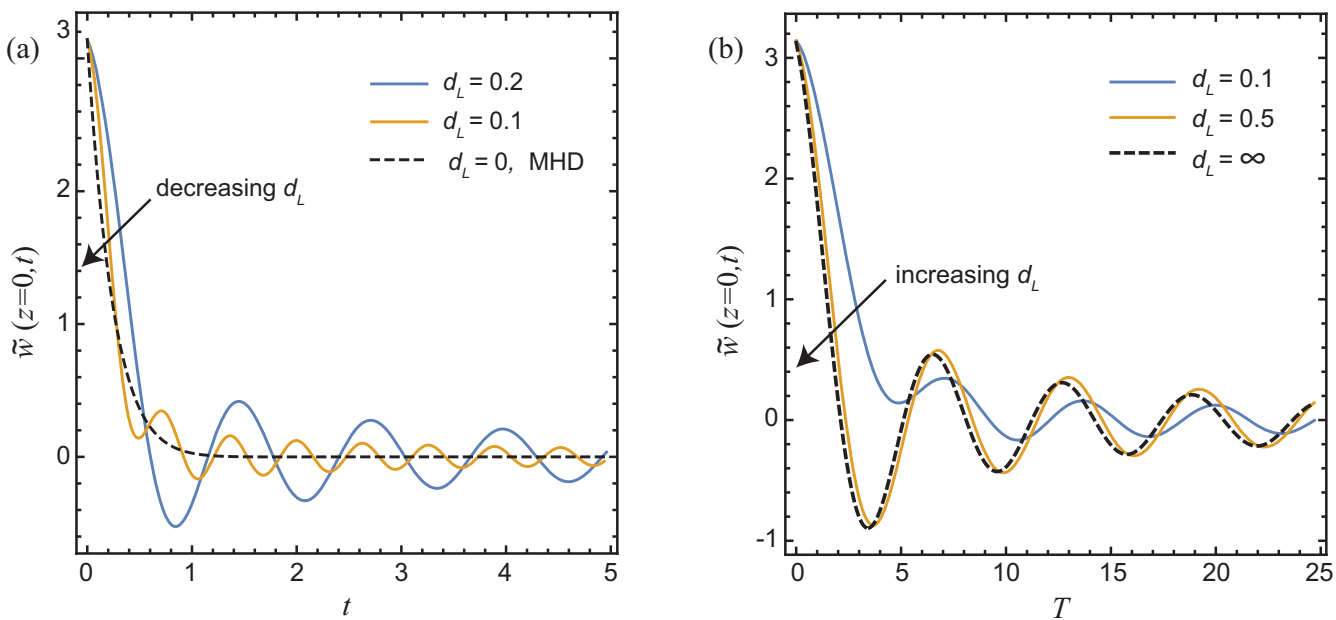

FIG. 2. Growth rate of the density interface perturbation, $d \eta / d t=\tilde{w}(0)$, as a function of the Larmor radius $d_{L}$ : (a) convergence towards the MHD limit $\left(d_{L}=0\right.$, dashed) as $d_{L}$ decreases, represented in original time $t$, and (b) convergence towards the large $d_{L}$ asymptote (dashed) as $d_{L}$ increases, represented in the rescaled time $T=t / d_{L}$. In both cases, the energy ratio and the Atwood number are fixed at $\beta=2$ and $\mathcal{A}=0.5$, respectively.

It is noted that since $\eta_{0}$ appears as a common factor of all quantities of interest, unity is assumed for its value in all the subsequent numerical results without loss of generality. Further, representative results are shown for the case $\rho_{2}>\rho_{1}$, or equivalently $0<\mathcal{A}<1$, in the following.

\section{A. IICE results}

We first establish the characteristic properties of the incompressible ion, compressible electron (IICE) Hall-MHD description by presenting an illustrative solution in comparison with its various limiting cases, including the regular MHD theory.

\section{Growth of the interface perturbation}

The interface perturbation growth is first shown to establish the suppression of RMI due to a perpendicularly applied magnetic field. Figure 2 illustrates the decaying growth rate of the density interface perturbation for all ranges of the Larmor radius $d_{L}$, from the MHD limit where $d_{L}=0$ is shown in (a), to the infinite $d_{L}$ limit shown in (b), with a fixed energy ratio $\beta$. The damped oscillation, analyzed in Sec. III B for large $d_{L}$, whose period depends on the ion cyclotron frequency, is also observed when $d_{L}$ is finite. This characteristic feature distinguishes the Hall-MHD equations from the regular MHD system, where fluctuations do not occur.

Integrating the growth rate $\tilde{w}$ over time leads to the magnitude of the interface perturbation, demonstrated in Fig. 3. When measured over the original time scale $t$, the effect of finite $d_{L}$ to the growth of the density interface perturbation is twofold, as shown in Fig 3(a). First, it causes oscillations of increasing frequencies as $d_{L}$ decreases, provided that $d_{L}>0$, rendering the MHD description, which exhibits monotonic growth, a singular limit as $d_{L} \rightarrow 0$. Second, interface growth in the Hall-MHD model still saturates in the long term, as in the MHD case, but now to a level that increases without bound with increasing $d_{L}$.

More revealing observations are made when the rescaled units for time and length are used, as given in Fig. 3(b). It is evident that the oscillation frequencies collapse to a state dictated by the ion cyclotron, extrapolating the large $d_{L}$ analysis as a convergent limit for increasing $d_{L}$ (see Sec. III B 3). 

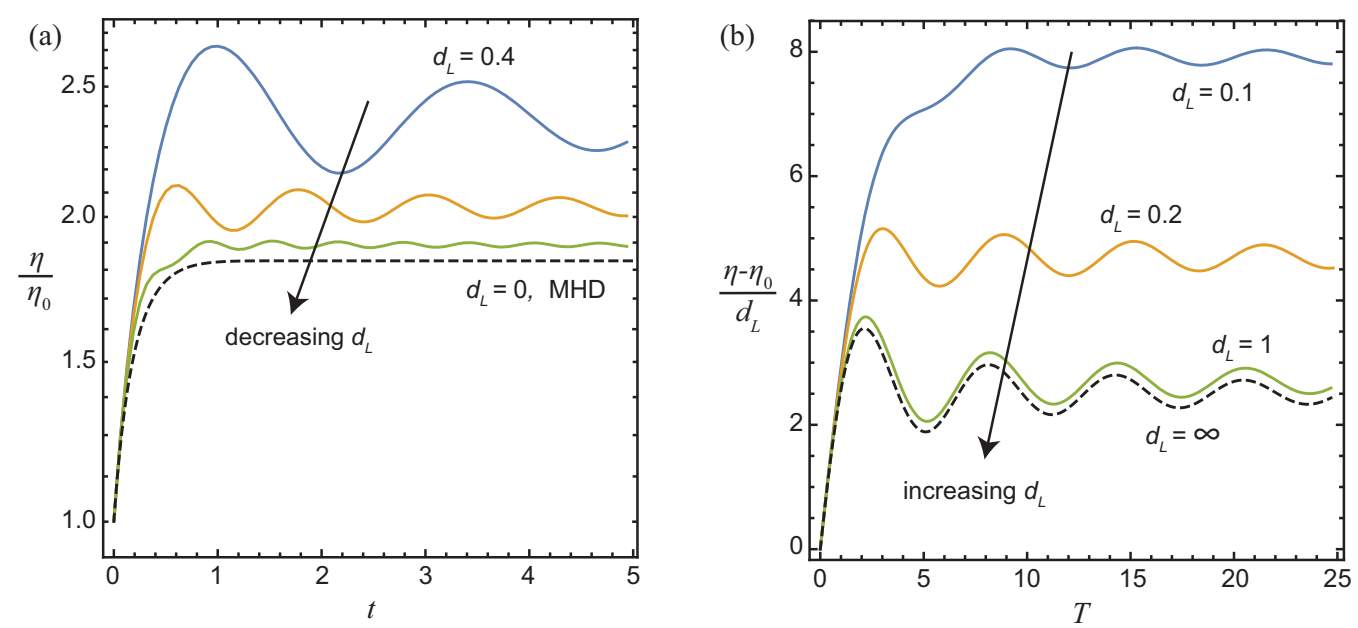

FIG. 3. Amplitude of the interface perturbation as a function of the Larmor radius $d_{L}$, in the incompressible ion compressible electron (IICE) Hall-MHD model. In (a), the original time unit $t$ and length unit $\eta_{0}$ are used for decreasing values of $d_{L}=0.4,0.2,0.1$ (solid lines) and $d_{L}=0$ (the MHD limit, dashed line). In (b), rescaled time $T=t / d_{L}$ and length $\eta / d_{L}$ are used with $d_{L}$ varying from 0.1 to infinity (dashed). For all series, the energy ratio and the Atwood number are fixed at $\beta=2$ and $\mathcal{A}=0.5$, respectively.

Additionally, Figs. 2(b) and 3(b) both confirm that the Hall-MHD model is most sensitive to the Larmor radius only when the ion skin depth is small, i.e., $0<d_{S} \lesssim 1$. Therefore, the large $d_{L}$ asymptotes obtained in Eqs. (54) and (56) are very good approximations if the Larmor radius significantly exceeds the initial perturbation wavelength for a fixed energy ratio $\beta$.

(a)

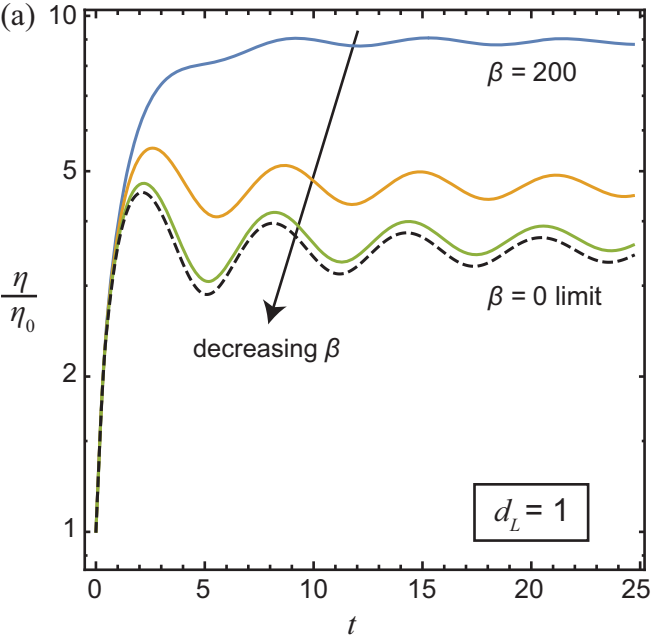

(b)

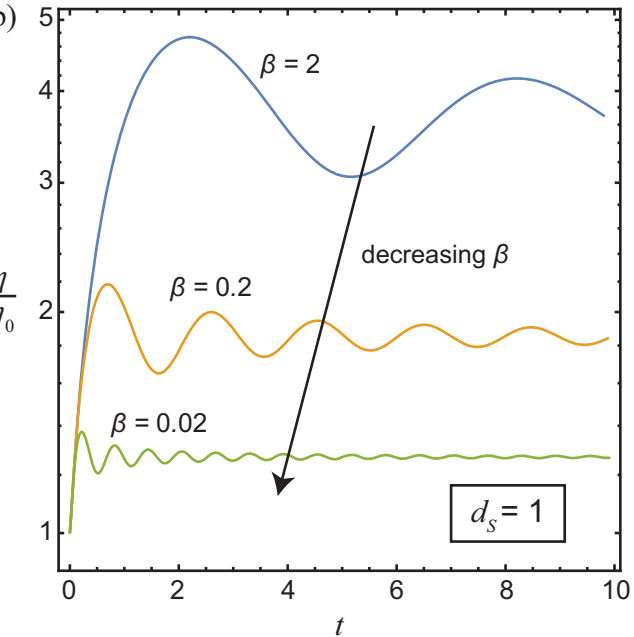

FIG. 4. Amplitude of the interface perturbation as a function of energy ratio $\beta$ for (a) fixed Larmor radius $d_{L}=1$ and (b) fixed ion skin depth $d_{S}=1$. The IICE model with an Atwood number of $\mathcal{A}=0.5$ is used throughout. Convergence towards the small $\beta$ limit (dashed line) is shown with decreasing $\beta=200,20,2$ (solid lines) in (a), whereas convergence towards zero interface growth is shown by further reducing $\beta=$ $2,0.2,0.02$ in (b). 

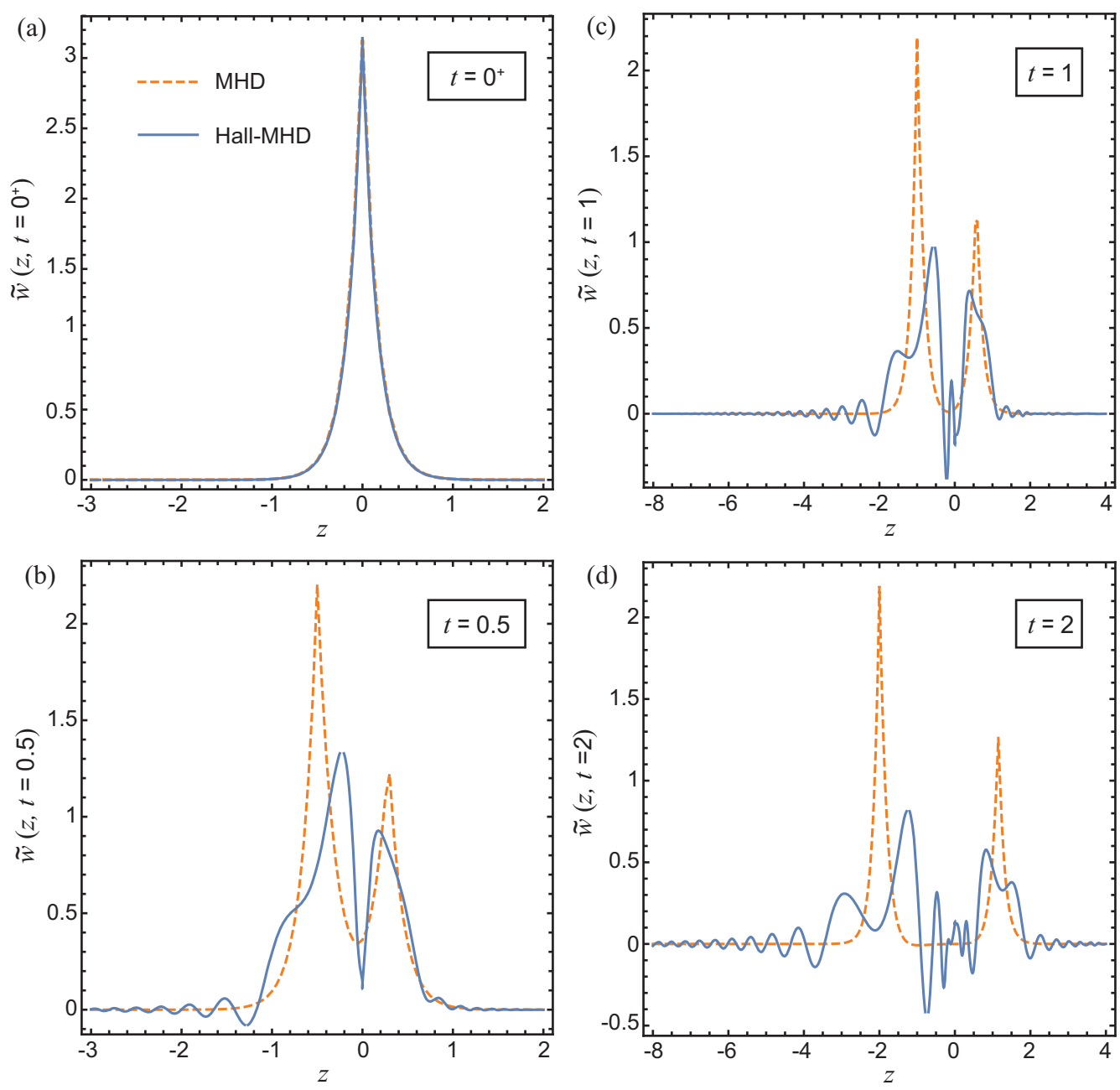

FIG. 5. Normal velocity profile from the IICE model, $\tilde{w}(z, t)$, for $t=0^{+}, 0.5,1,2$, in (a)-(d), respectively. The Hall-MHD solutions, obtained for $\beta=2, d_{L}=d_{S}=0.1$, and $\mathcal{A}=0.5$, given by the solid lines, are compared to the regular MHD solutions of the same energy ratio and Atwood number, shown as dashed lines.

Next, we illustrate in Fig. 4 the effect of changing energy ratio $\beta$ on the interface growth. This is performed with $d_{L}$ fixed in 4(a) and $d_{S}$ fixed in 4(b). The former may be physically achieved by decreasing the upstream fluid density and the latter by increasing the applied magnetic field strength. In both cases, as in the MHD study [14], increasing $\beta$ in the Hall-MHD solution ultimately recovers the hydrodynamic RMI growth that is unbounded. However, the effect of decreasing $\beta$ differs in the two cases shown. When the Larmor radius $d_{L}$ is fixed, the interface growth is bounded below by the limiting $\beta=0, d_{S}=\infty$ asymptote given in (56), where partial suppression of the RMI, predicted by Eq. (57) in the long term, is achieved at most, whereas, when the ion skin depth $d_{S}$ is fixed, complete suppression of the RMI occurs at a rate the same as in MHD, i.e., $\eta(\infty) / \eta_{0} \sim O\left(\beta^{1 / 2}\right)$, while the oscillation frequency increases proportional to $d_{L} \propto \beta^{-1 / 2}$.

\section{Velocity profile}

Away from the interface, the normal flow velocity profile $\tilde{w}(z, t)$ is compared between the HallMHD and regular MHD models directly in Fig. 5, where the evolution of the velocity profile is 


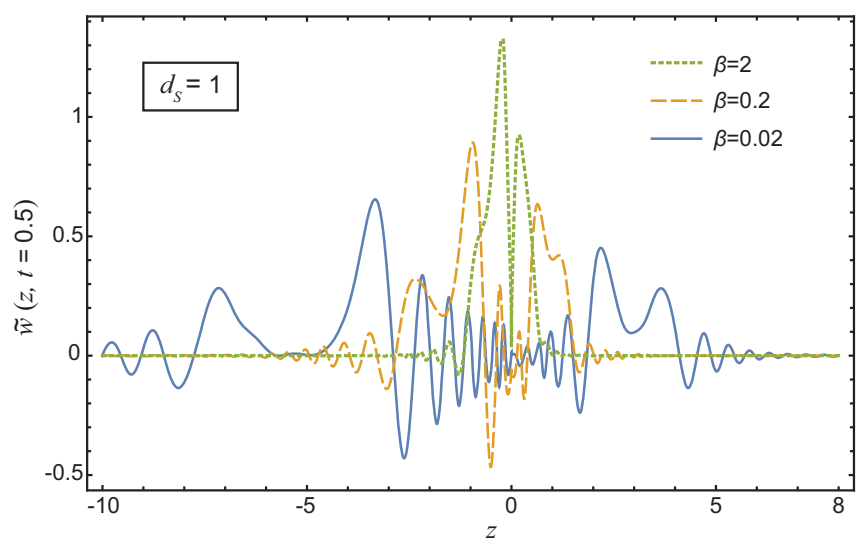

FIG. 6. Normal velocity profiles from the IICE model, $\tilde{w}(z, t=0.5)$, for increasing strength of the applied magnetic field, with varying $\beta=2,0.2,0.02$ and constant $d_{S}=1, \mathcal{A}=0.5$.

shown at four sequential instants of time. At $t=0^{+}$, the initial velocity distribution resulting from the impulsive acceleration is identical for the Hall-MHD and MHD systems, a purely hydrodynamic result, as shown in Fig. 5(a). Nonetheless, owing to its dispersive nature [19], the propagation of the initial impulse in the Hall-MHD system, demonstrated in Figs. 5(b)-5(d) for $t>0$, exhibits highly oscillatory patterns along the normal axis, which differs markedly from the MHD prediction where the Alfvén waves are responsible for carrying away the velocity peak in each fluid region $[14,15]$. Furthermore, it is clear that the fast waves associated with the Hall-MHD model are able to produce rotational flow ahead of the Alfvén wave fronts, which limits the propagation of information in the MHD description.

Increasing the applied magnetic field strength in this case has a twofold effect on stabilizing the interface growth, as shown in Fig. 6, where three velocity profiles are compared at one instance for different values of $\beta$. As $\beta$ decreases, first, oscillations of the interface growth rate after the decay

(a)

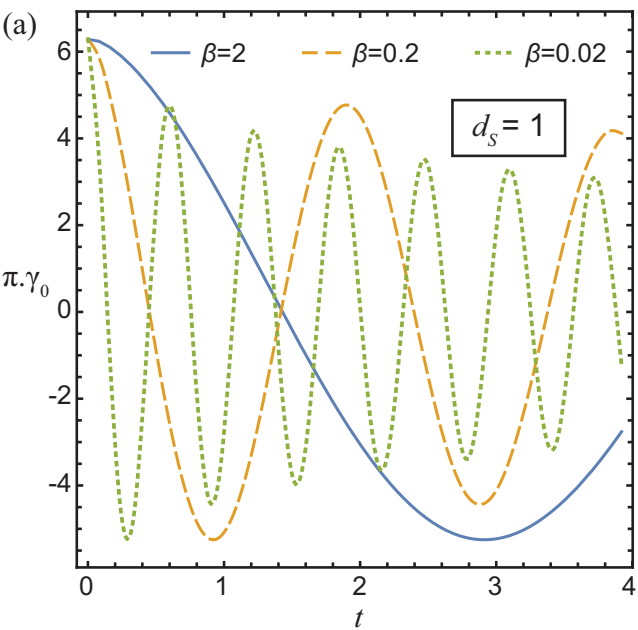

(b)

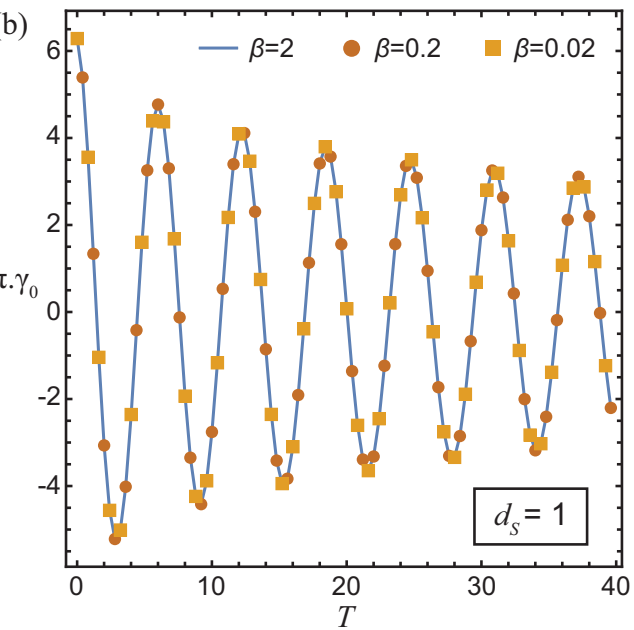

FIG. 7. Effect of increasing applied magnetic field in the IICE model on the circulation deposition at the interface, $\gamma_{0}$, as $\beta$ is decreased using $\beta=2,0.2,0.02$ with constant $d_{S}=1, \mathcal{A}=0.5$ over (a) the original time scale $t$ and (b) the cyclotron time scale $T=t / d_{L}$. 

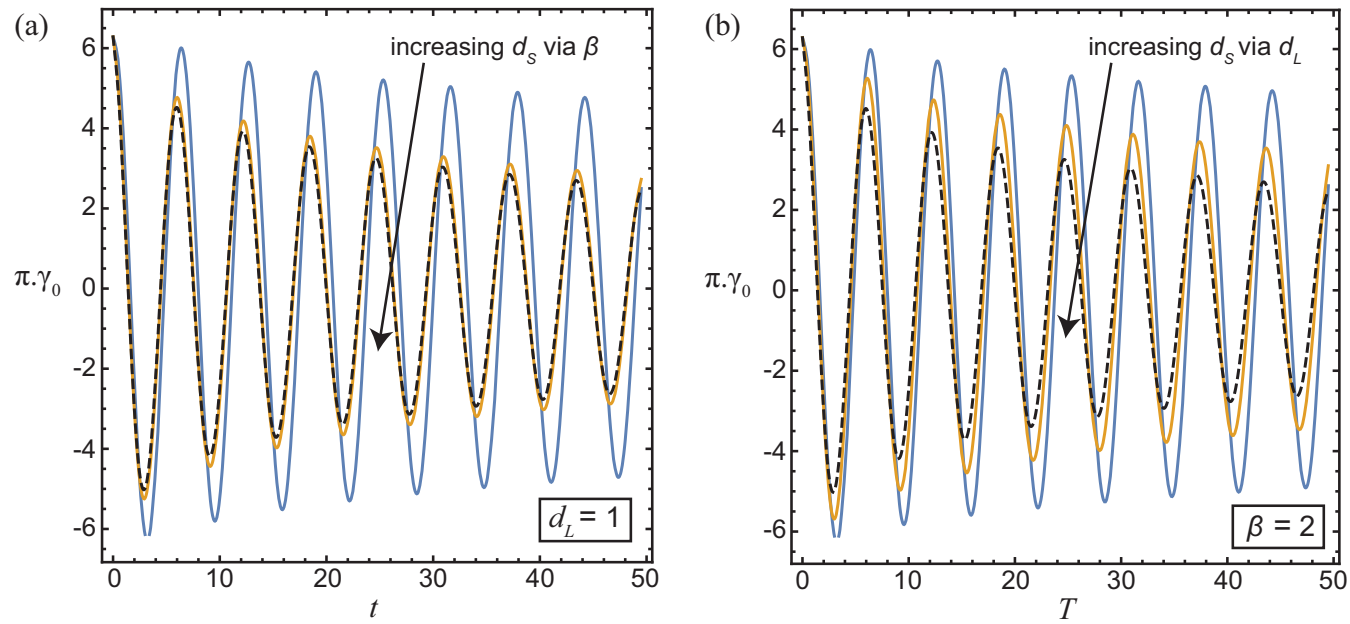

FIG. 8. Effect of increasing ion skin depth in the IICE model on the circulation deposition at the interface, $\gamma_{0}$, for constant $\mathcal{A}=0.5$. (a) Increase $d_{S}$ via decreasing energy ratio $\beta=100,2$, 0 , while holding $d_{L}=1$ fixed. (b) Increase $d_{S}$ via increasing Larmor radius $d_{L}=0.1,0.5, \infty$, in rescaled time $T=t / d_{L}$, while holding $\beta=2$ fixed. In both cases, the dashed line represents the same asymptotic limit of $d_{S}=\infty$.

of the initially impulsively generated peak are suppressed; second, all of the induced disturbances are carried away at a faster speed by the Alfvén waves in both upstream and downstream flows [see Eq. (45)].

\section{Circulation and vorticity}

We now explore the IICE Hall-MHD model distribution of circulation and vorticity. First, as discussed in Sec. III A, the discontinuity of tangential velocity across the density interface gives rise to a vortex sheet where circulation is deposited, contrasting the regular MHD model that supports a no-slip condition across the interface. The evolution of the accumulated circulation, $\gamma_{0}$, derived from Eq. (40), is plotted for decreasing $\beta$ with $d_{S}$ fixed using both time scales $t$, and $T$, in Fig. 7. Over the original time scale $t$, decreasing $\beta$ captures more cycles of decaying oscillations over a finite initial window of time, so that in the $\beta \rightarrow 0$ limit all fluctuations of finite amplitude are packed into an infinitesimally short period of time immediately after the initial impulse, leaving $\gamma_{0}=0$ for all $t>0^{+}$. This is an example of nonuniform convergence at $t=0$. More is revealed when the scaled time $T$ is used in 7(b): curves obtained for different $\beta$ all seem to collapse. This is indeed expected because substituting $\beta=2 d_{L}^{2} / d_{S}^{2}$ into (40) yields an expression of the form $\Gamma_{0} / d_{L}=f\left(d_{S} ; \sigma\right)$, where the known function $f$ does not depend on $\beta$. Therefore, this universal curve for all $\beta>0$ encodes the entire decaying history of $\gamma_{0}$ over the infinitesimal time window of $t$ as $\beta \rightarrow 0$ shown in 7(a).

Having established that the shape of $\gamma_{0}(T)$ only depends on $d_{S}$ (for a fixed $\mathcal{A}$ ), Fig. 8 plots its convergence to the $d_{S} \rightarrow \infty$ limit obtained in (53) through either decreasing $\beta$ as presented in (a) or increasing $d_{L}$ as depicted in (b). It is clear that the decaying rate of the oscillation envelope is maximized at the limiting solution. Nevertheless, as in the case for the interface growth presented earlier, the convergence of circulation deposition towards the large $d_{S}$ limiting value is fast. For moderate values of $\beta$ and $d_{L}$, the asymptotic limit provides reasonable approximation to the true solution.

Away from the interface, because the $y$ component of vorticity in the continuous flow domain is governed by the forced wave equation [see Eq. (45)], rotational flow is no longer confined to a region defined by the two Alfvén wave fronts in each fluid, as in the MHD system. This is demonstrated in Fig. 9, where the vorticity profile excluding $z=0$ is plotted for two instants of time. 


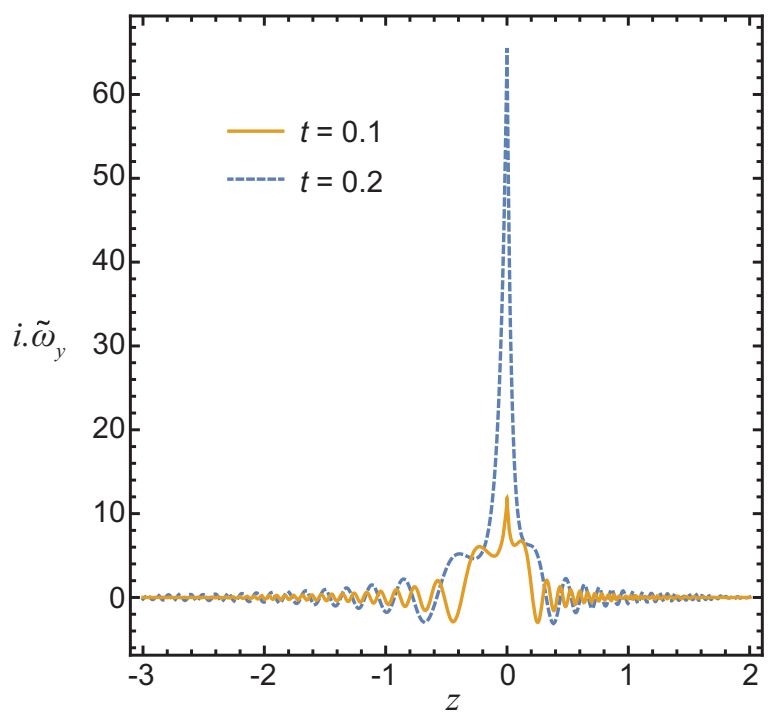

FIG. 9. Vorticity profile $\tilde{\omega}_{y}(z, t)$ at two time instants obtained using the IICE model: $t=0.1$ (solid) and $t=0.2$ (dashed). Both solutions are obtained for $d_{L}=0.1, \beta=2$, and $\mathcal{A}=0.5$.

Therefore, the total circulation in the $x, z$ plane, calculated by integrating the vorticity component $\omega_{y}$ over the infinite band $\{(x, z): z \in \mathbb{R}, x \in(0, \Lambda / 2)\}$, given by

$$
\gamma(t)=\gamma_{0}(t)+\frac{i}{\pi} \int_{\mathbb{R} \backslash\{0\}} \tilde{\omega}_{y}(z, t) d z
$$

is nonconservative over time. For example, as seen in Figs. 8(b) and 9, it is numerically verified that $\gamma(0.2)>\gamma(0.1)$, implying that circulation is generated by the magnetic forcing during that
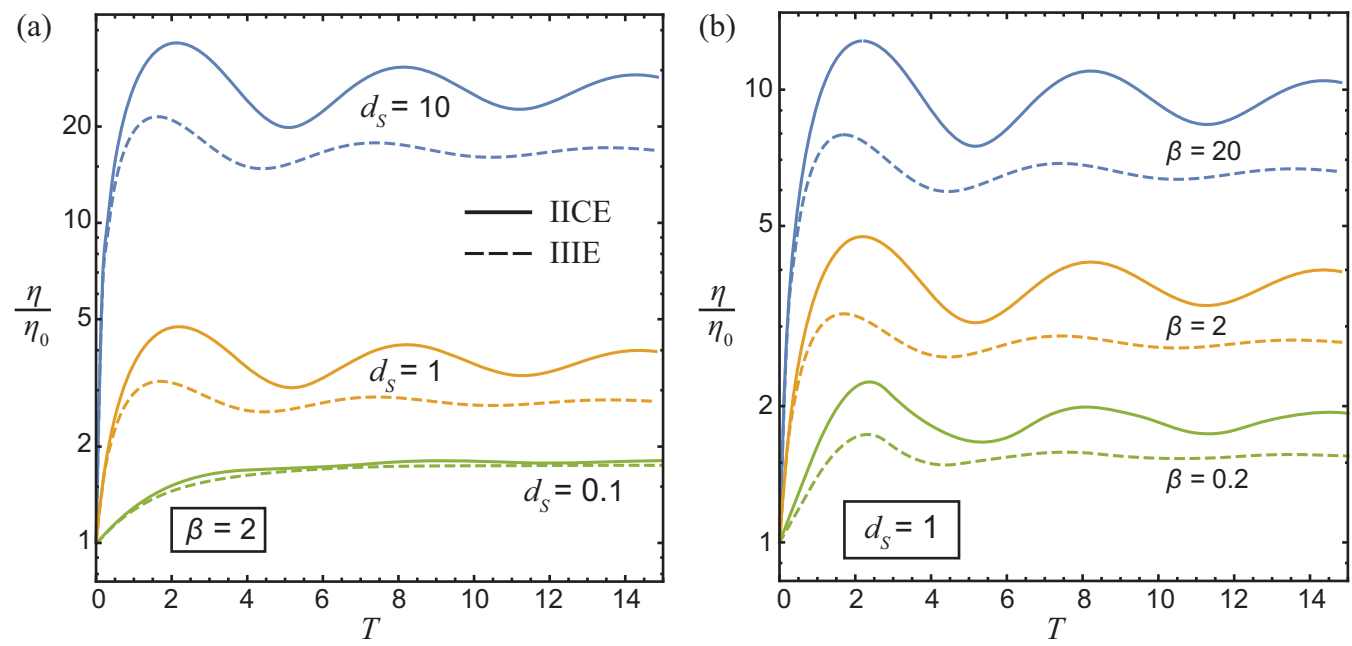

FIG. 10. Amplitude growth of the interface perturbation, $\eta / \eta_{0}$, predicted by the IICE model (solid lines) and the IIIE model (dashed lines). Comparisons are made for varying $d_{S}=0.1,1,10$, with $\beta=2$ fixed in (a), and for changing $\beta=0.2,2,20$, with $d_{S}=1$ fixed in (b). All series are obtained in the cyclotron time scale $T$ for fluids with $\mathcal{A}=0.5$. 

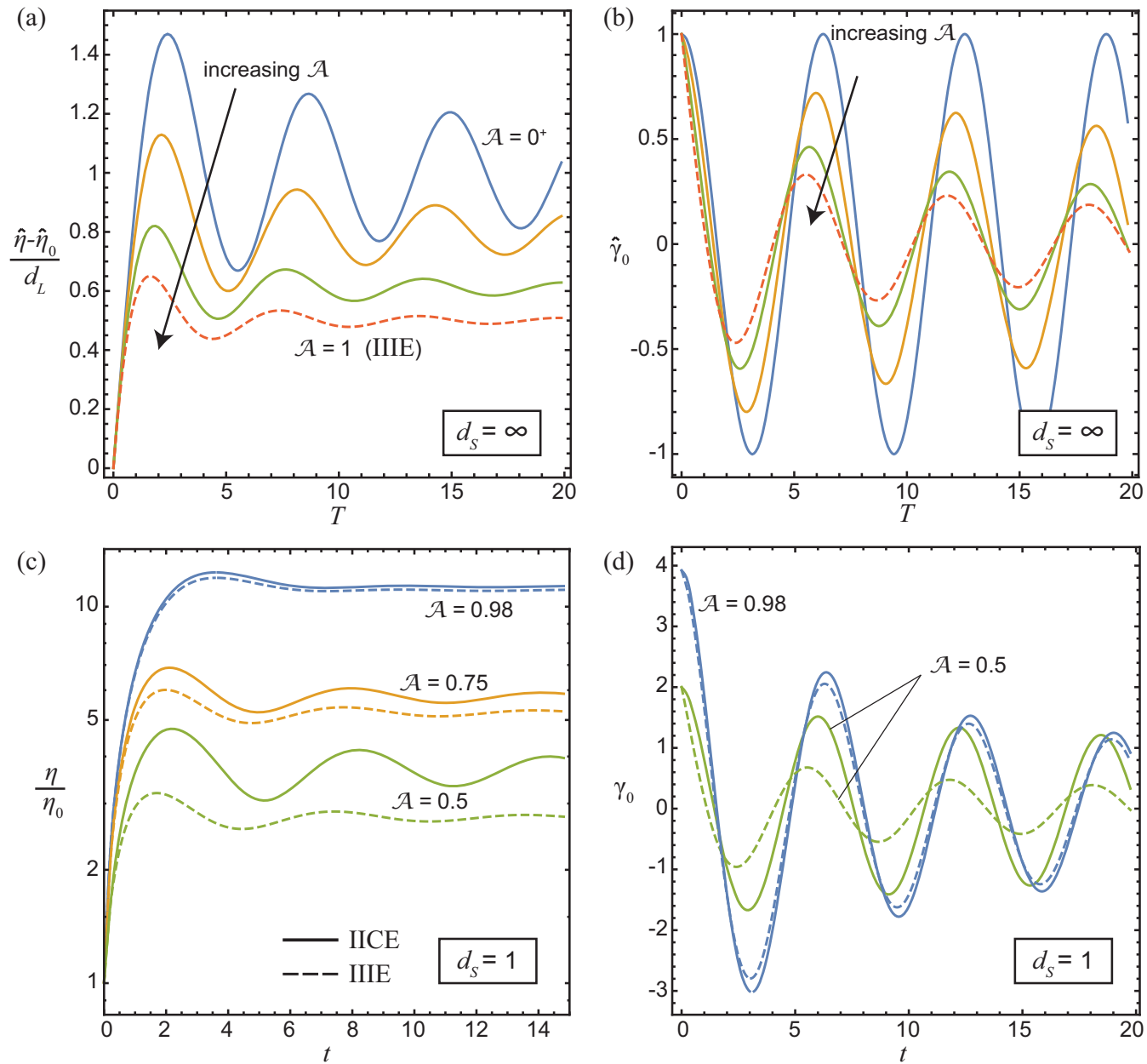

FIG. 11. Convergence of interface statistics obtained from the IICE model (solid lines), towards those of the IIIE model (dashed lines), as $\mathcal{A} \rightarrow 1$. The $d_{S}=\infty$ asymptotic behavior of (a) the renormalized interface growth $\hat{\eta}$ and (b) the renormalized circulation deposition $\hat{\gamma}_{0}$ are plotted as functions of increasing $\mathcal{A}=0,0.5,0.8,1$. Similar comparisons are made for finite $d_{S}=1$ corresponding solutions for $\eta$ and $\gamma_{0}$ in (c) and (d), respectively. $\beta=2$ is used throughout.

period of time. It is stressed that the total circulation collects a point mass contribution $\gamma_{0}$ at the interface $z=0$, at any instant. In comparison, the regular MHD model cannot sustain circulation at the interface, nor does it inject additional vorticity into the flow after the initial baroclinic generation.

\section{B. IIIE results and model comparison}

Finally, we show results obtained using the incompressible ion incompressible electron (IIIE) Hall-MHD model and make a comparison against the IICE system to show that the key features are qualitatively similar in both models.

The growth of the interface amplitude predicated by the two models is first illustrated in Fig. 10 as a function of $d_{S}$ and $\beta$, where the cyclotron frequency rescaling applies effectively in both cases. It is seen that the IIIE model consistently gives a smaller interface growth, through fluctuations whose amplitude dampens significantly quicker over each cycle. These differences however diminish as 

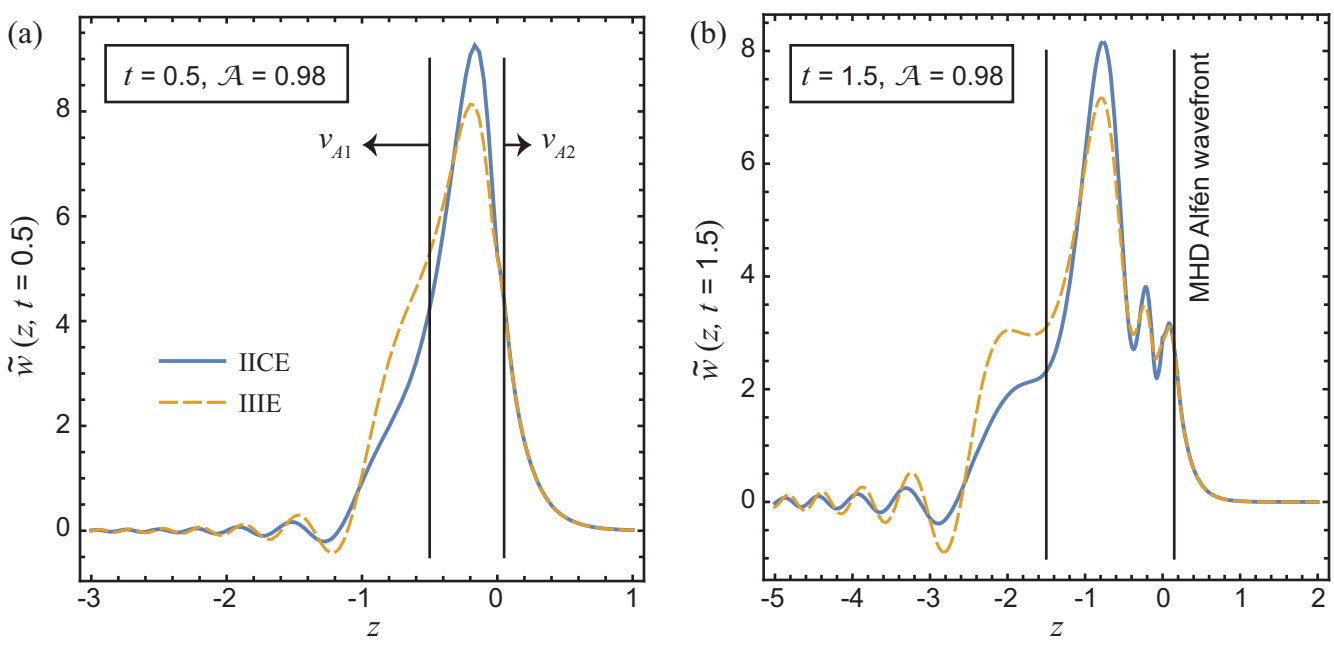

FIG. 12. Comparison of normal velocity profile between the IICE (solid line) and IIIE (dashed line) flow models for high density ratio, $\mathcal{A}=0.98$, at time $t=0.5$ in (a) and $t=1.5$ in (b). $d_{S}=0.1$ and $\beta=2$ are held fixed in all cases. The vertical lines indicate the position of Alfén wave fronts associated with the MHD model traveling at speed $v_{A_{i}}$ in the fluid region $i=1,2$, left or right to the interface.

either $d_{S}$ or $\beta$ is decreased, as both models converge to the respective common limit of MHD theory, or the no-motion solution due to strong magnetic field.

In Fig. 11, a series of the interface characteristics obtained from both systems are shown to converge as the Atwood number $\mathcal{A}$ approaches unity. Figures 11(a) and 11(b) evidence the analysis of Sec. IV $\mathrm{C}$ that, in the large $d_{S}$ limit, the IICE and IIIE models coincide when $\mathcal{A}=1$. This property is extrapolated to hold for finite $d_{S}$ as well in Figs. 11(c) and 11(d), where the interface amplitude $\eta$ and circulation deposition $\gamma_{0}$ are shown to be dependent on $\mathcal{A}$ in both models, however with their difference diminishing as $\mathcal{A}$ is increased. It should also be clarified that particularly $\eta$ is an increasing function of $\mathcal{A}$, as shown in 11(c), which does not contradict 11(a) where the renormalized $\hat{\eta}$ is plotted in order to illustrate the full range of variability.

Nevertheless, the convergence of interface statistics between the IICE and IIIE models as $\mathcal{A}$ increases does not generalize to the continuous regions of the flow, for finite $d_{S}$. This is demonstrated in Fig. 12, where the normal velocity profile $\tilde{w}(z)$ is computed at a high Atwood number $\mathcal{A}=0.98$ (equivalently $\rho_{2} / \rho_{1}=100$ ) and $d_{S}=1$, for both models. The vorticity transport in the light fluid side (left) is clearly different between the two models in a region close to the corresponding Alfvén wave front. The heavy fluid side, however, including the neighborhood of the interface, appears insensitive to the model used for the electrons.

\section{CONCLUSIONS}

We have examined the behavior of an impulsively accelerated perturbed interface separating conducting fluids of different densities, in the presence of a magnetic field that is parallel to the acceleration, using the Hall-MHD equations. Assuming the ions form an incompressible fluid, two impulsive models are proposed, first with a compressible electron flow and second with an incompressible electron flow. These models are analytically approached as linearized initial-value problems that accommodate all three components of the velocity and magnetic fields. By applying the appropriate jump conditions across contact discontinuity for each model, the resulting flow fields demonstrate that, in both cases, the growth of the interface perturbation is limited by the imposed magnetic field, establishing suppression of the RMI when the ion skin depth (or the Larmor radius) is significant compared to the perturbation wavelength. In such flow regimes inaccessible to the ideal 
MHD theory, oscillations associated with the ion cyclotron effect are imposed onto the entire flow domain, substantially altering the vorticity dynamics displayed by the ideal MHD model that drives the suppression of the RMI. Most significantly, the jump conditions in the Hall-MHD case permit circulation deposition on the interface, which is precluded in ideal MHD when a magnetic field penetrates the interface. Together with a continuous vorticity production away from the interface due to the magnetic contribution to the vorticity equation, the total circulation in the domain is no longer a conserved quantity. Furthermore, the dispersive nature of the Hall-MHD equations also drastically affects the transport of vorticity, which is no longer confined to the Alfvén fronts propagating outwards from the interface, as in the ideal MHD case.

By varying the nondimensional Larmor radius, the ion skin depth, the energy ratio $(\beta)$, and the fluid density ratio (or the Atwood number), it is found that the hydrodynamic RM flow limit is recovered when $\beta$ is large and the MHD limit is attained when $d_{S}$ (or $d_{L}$ ) is small. Further there exists a large $d_{S}$ limit that is opposite to the MHD system, where solutions at the interface converge rapidly for $d_{S}$ greater than order unity. Lastly, the two somewhat different models (IICE and IIIE) are compared to show qualitatively similar results across the entire parameter space.

\section{ACKNOWLEDGMENT}

This work was supported by the KAUST Office of Sponsored Research under Award No. $\mathrm{URF} / 1 / 3418-01$.

[1] G. H. Markstein, Flow disturbances induced near a slightly wavy contact surface, or flame front, traversed by a shock wave, J. Aeronautical Sci. 24, 238 (1957).

[2] R. D. Richtmyer, Taylor instability in shock acceleration of compressible fluids, Commun. Pure Appl. Math. 13, 297 (1960).

[3] E. E. Meshkov, Instability of the interface of two gases accelerated by a shock wave, Fluid Dyn. 4, 101 (1969).

[4] D. Arnett, The role of mixing in astrophysics, Astrophys. J., Suppl. Ser. 127, 213 (2000).

[5] A. M. Khokhlov, E. S. Oran, and G. O. Thomas, Numerical simulation of deflagration-to-detonation transition: The role of shock-flame interactions in turbulent flames, Combust. Flame 117, 323 (1999).

[6] J. Yang, T. Kubota, and E. E. Zukoski, Applications of shock-induced mixing to supersonic combustion, AIAA J. 31, 854 (1993).

[7] M. Brouillette, The Richtmyer-Meshkov instability, Annu. Rev. Fluid Mech. 34, 445 (2002).

[8] J. D. Lindl, R. L. McCrory, and E. M. Campbell, Progress toward ignition and burn propagation in inertial confinement fusion, Phys. Today 45(9), 32 (1992).

[9] M. J.-E. Manuel, C. K. Li, F. H. Séguin, J. Frenje, D. T. Casey, R. D. Petrasso, S. X. Hu, R. Betti, J. D. Hager, D. D. Meyerhofer et al., First Measurements of Rayleigh-Taylor-Induced Magnetic Fields in Laser-Produced Plasmas, Phys. Rev. Lett. 108, 255006 (2012).

[10] F. H. Séguin, C. K. Li, M. J.-E. Manuel, H. G. Rinderknecht, N. Sinenian, J. A. Frenje, J. R. Rygg, D. G. Hicks, R. D. Petrasso, J. Delettrez et al., Time evolution of filamentation and self-generated fields in the coronae of directly driven inertial-confinement fusion capsules, Phys. Plasmas 19, 012701 (2012).

[11] I. V. Igumenshchev, A. B. Zylstra, C. K. Li, P. M. Nilson, V. N. Goncharov, and R. D. Petrasso, Selfgenerated magnetic fields in direct-drive implosion experiments, Phys. Plasmas 21, 062707 (2014).

[12] D. Bond, V. Wheatley, R. Samtaney, and D. I. Pullin, Richtmyer-Meshkov instability of a thermal interface in a two-fluid plasma, J. Fluid Mech. 833, 332 (2017).

[13] R. Samtaney, Suppression of the Richtmyer-Meshkov instability in the presence of a magnetic field, Phys. Fluids 15, L53 (2003). 
[14] V. Wheatley, D. I. Pullin, and R. Samtaney, Stability of an Impulsively Accelerated Density Interface in Magnetohydrodynamics, Phys. Rev. Lett. 95, 125002 (2005).

[15] V. Wheatley, R. Samtaney, and D. I. Pullin, The Richtmyer-Meshkov instability in magnetohydrodynamics, Phys. Fluids 21, 082102 (2009).

[16] J. Cao, Z. Wu, H. Ren, and D. Li, Effects of shear flow and transverse magnetic field on RichtmyerMeshkov instability, Phys. Plasmas 15, 042102 (2008).

[17] V. Wheatley, R. Samtaney, D. I. Pullin, and R. M. Gehre, The transverse field Richtmyer-Meshkov instability in magnetohydrodynamics, Phys. Fluids 26, 016102 (2014).

[18] B. Srinivasan and X.-Z. Tang, Mechanism for magnetic field generation and growth in Rayleigh-Taylor unstable inertial confinement fusion plasmas, Phys. Plasmas 19, 082703 (2012).

[19] N. Shen, Y. Li, D. I. Pullin, R. Samtaney, and V. Wheatley, On the magnetohydrodynamic limits of the ideal two-fluid plasma equations, Phys. Plasmas 25, 122113 (2018).

[20] A. L. Ortega, D. J. Hill, D. I. Pullin, and D. I. Meiron, Linearized Richtmyer-Meshkov flow analysis for impulsively accelerated incompressible solids, Phys. Rev. E 81, 066305 (2010).

[21] E. Hameiri, Generalized shock conditions and the contact discontinuity in the Hallmagnetohydrodynamics model, Phys. Plasmas 20, 022112 (2013).

[22] P. Rosenau, J. A. Tataronis, and G. Conn, Elements of magnetohydrodynamics with the Hall current. Part 1. Nonlinear phenomena, J. Plasma Phys. 21, 385 (1979).

[23] P. M. Bellan, Fundamentals of Plasma Physics (Cambridge University Press, Cambridge, UK, 2008).

[24] E. Hameiri, A. Ishizawa, and A. Ishida, Waves in the Hall-magnetohydrodynamics model, Phys. Plasmas 12, 072109 (2005).

[25] M. Abramowitz and I. A. Stegun, Handbook of Mathematical Functions: With Formulas, Graphs, and Mathematical Tables (Courier Corporation, New York, 1965), Vol. 55.

[26] J. Abate and P. P. Valkó, Multi-precision Laplace transform inversion, Int. J. Numer. Methods Eng. 60, 979 (2004).

[27] A. Talbot, The accurate numerical inversion of Laplace transforms, IMA J. Appl. Math. 23, 97 (1979). 